\title{
Cubic spline fractal solutions of two-point boundary value problems with a non-homogeneous nowhere differentiable term
}

\author{
A.K.B. Chand ${ }^{a}$, K.R. Tyada ${ }^{a}$, M.A. Navascués ${ }^{b, *}$ \\ a Department of Mathematics, Indian Institute of Technology Madras, Chennai 600036, India \\ b Departmento de Matemática Aplicada, Escuela de Ingeniería y Arquitectura, Universidad de Zaragoza-50018, Spain
}

\section{A R T I C L E I N F O}

\section{Article history:}

Received 9 January 2020

Received in revised form 15 October 2020

\section{MSC:}

65D05

65D07

$65 \mathrm{~L} 10$

$65 \mathrm{~L} 20$

26A27

$28 \mathrm{~A} 80$

\section{Keywords:}

Splines

Fractal interpolation

Ordinary differential equations

Boundary value problems

Nondifferentiability

Fractals

\begin{abstract}
A B S T R A C T
Fractal interpolation functions (FIFs) supplement and subsume all classical interpolants. The major advantage by the use of fractal functions is that they can capture either the irregularity or the smoothness associated with a function. This work proposes the use of cubic spline FIFs through moments for the solutions of a two-point boundary value problem (BVP) involving a complicated non-smooth function in the non-homogeneous second order differential equation. In particular, we have taken a second order linear BVP: $y^{\prime \prime}(x)+Q(x) y^{\prime}(x)+P(x) y(x)=R(x)$ with the Dirichlet's boundary conditions, where $P(x)$ and $Q(x)$ are smooth, but $R(x)$ may be a continuous nowhere differentiable function. Using the discretized version of the differential equation, the moments are computed through a tridiagonal system obtained from the continuity conditions at the internal grids and endpoint conditions by the derivative function. These moments are then used to construct the cubic fractal spline solution of the BVP, where the non-smooth nature of $y^{\prime \prime}$ can be captured by fractal methodology. When the scaling factors associated with the fractal spline are taken as zero, the fractal solution reduces to the classical cubic spline solution of the BVP. We prove that the proposed method is convergent based on its truncation error analysis at grid points. Numerical examples are given to support the advantage of the fractal methodology.
\end{abstract}

(c) 2020 Elsevier B.V. All rights reserved.

\section{Introduction}

Differential equations have been used to describe or model real life phenomena. They occur frequently in many areas of scientific and engineering disciplines like physics, chemistry, architecture, ecology, chemical kinetics, mechanical engineering, quantum mechanics, electrical engineering, civil engineering, meteorology, and a relatively new science called chaos. The ultimate goal of solving a differential equation is finding an explicit or an analytical solution which can be used to describe the underlying physical phenomena or scientific experiment.

On the other hand, many of the real world and experimental signals are irregular and rarely render a sensation of smoothness in their structures. Thereby traditional interpolants with simple geometric structures may not describe these fine microscopic patterns effectively. Thus, there is a need for the search of interpolation techniques which can produce interpolants that fail to be differentiable in a dense subset of the interpolation interval. To address this cause, Barnsley [1] introduced the notion of fractal interpolation using the theory of iterated function system. Graph of the fractal

\footnotetext{
* Corresponding author.

E-mail addresses: chand@iitm.ac.in (A.K.B. Chand), kurmaths86@gmail.com (K.R. Tyada), manavas@unizar.es (M.A. Navascués).
} 
interpolant possesses a non-integer dimension which can be used as a quantifier for the complexity of the underlying phenomenon. Barnsley and Harrington [2] initiated the construction of smooth FIFs, and unfolded the notable relationship between fractal functions and splines. Smooth FIFs can be applied to generalize the classical interpolation techniques (see, for instance, [3-9]). Therefore, fractal interpolation offers the flexibility of choosing either a smooth or non-smooth interpolant depending on the modeling problem at hand.

A boundary value problem (BVP) is a differential equation together with a set of additional constraints called the boundary conditions. A solution to a boundary value problem satisfies the differential equation and the prescribed boundary conditions. There are many methods developed in the literature for solving higher order BVPs. Bickley [10] used general cubic splines and Albasiny [11] used cubic splines through moments for the solution of linear (regular) two-point BVPs. Fyfe [12] discussed the application of deferred corrections to the method proposed by Bickley. Jain and Aziz [13] proposed a parametric spline function consisting of trigonometric and polynomial maps of order 1 for the solution of the system of ordinary and partial differential equations. Al-Said [14] presented a method to approximate the solution of linear second order BVP and also to determine first, second and third order derivatives at every point of the range of integration. Ramos and others [15-17] used different types of block methods to the direct approximation of the solution of fourth order Boundary Value Problems. Recently, differential or integral equation is discretized by using a novel fractional step in [18].

Sometimes variables representing the derivatives may be irregular in various physical phenomena. For examples: (i) a sphere falling in a wormlike micellar solution undergoes continual oscillations as it falls [19] (ii) the motion of a pendulum on a cart has varying irregularity in the study of acceleration (2nd derivative) in nonlinear control systems [20]. Therefore, if we can model the motion of these phenomena by using 2nd order ODE with certain boundary conditions, then use of fractal splines may be advantageous to capture the irregularity/fractality associated with the derivative. Thus, we expect fractal spline solutions of BVP for these nonlinear phenomena.

In this paper, we consider a two point BVP whose solution is a curve obtained by a fractal methodology through discretization of the domain, and this solution is an extension of the cubic spline defined in Albasiny [11]. The classical cubic spline does not give appropriate solution if the non-homogeneous differential equation involves a continuous function but not differentiable in a dense subset of the interpolation interval. Since the classical cubic spline is a particular case of fractal spline, the proposed method can be used to consider any type of continuous function in a BVP. We define the prerequisite material on (smooth) fractal functions and the general two-point second order boundary value problem in Section 2. We develop the description of our methods to solve the two-point BVPs using the cubic spline FIFs through moments in Section 3. Truncation error analysis is studied in Section 4. Finally, the proposed methods are used to solve BVPs in Section 5.

\section{Preliminaries}

To equip ourselves with the requisite general material, we will discuss the construction of fractal functions in Section 2.1, and the basics of a 2nd order BVP in Section 2.2.

\subsection{Fractal interpolation function}

This section targets to equip a novice reader with the basics of fractal interpolation. These materials are collected from the well-known treatises $[1,2,21]$.

For $r \in \mathbb{N}$, denote $\mathbb{N}_{r}$ as the set of first $r$ natural numbers. For $N>1$, let $x_{0}<x_{1}<\cdots<x_{N}$ be real numbers and $I=\left[x_{0}, x_{N}\right]$. Let the prescribed set of interpolation data be $\left\{\left(x_{j}, y_{j}\right) \in I \times \mathbb{R}: j \in \mathbb{N}_{N} \cup 0\right\}$. We want to construct a finite number of contraction maps in $\mathbb{R}^{2}$ for an iterated function system (IFS) so that its fixed point is the required fractal function. The abscissa values are defined through $L_{i}$ and the ordinate values are defined through $F_{i}$ (see [1] for details). For $i \in \mathbb{N}_{N}$, set $I_{i}=\left[x_{i-1}, x_{i}\right]$ and let $L_{i}: I \rightarrow I_{i}$ be contractive homeomorphisms such that

$$
\left.\begin{array}{r}
L_{i}\left(x_{0}\right)=x_{i-1}, \quad L_{i}\left(x_{N}\right)=x_{i} \forall i \in \mathbb{N}_{N}, \\
\left|L_{i}(x)-L_{i}\left(x^{*}\right)\right| \leq l_{i}\left|x-x^{*}\right| \forall x, x^{*} \in I \text { for some } 0<l_{i}<1 .
\end{array}\right\}
$$

Let $F_{i}: I \times \mathbb{R} \rightarrow \mathbb{R}$ be continuous maps satisfying

$$
\left.\begin{array}{l}
F_{i}\left(x_{0}, y_{0}\right)=y_{i-1}, F_{i}\left(x_{N}, y_{N}\right)=y_{i}, i \in \mathbb{N}_{N}, \\
\left|F_{i}(x, y)-F_{i}\left(x, y^{\prime}\right)\right| \leq k_{i}\left|y-y^{\prime}\right|, x \in I ; y, y^{\prime} \in \mathbb{R} ; \text { for some } 0 \leq k_{i}<1 .
\end{array}\right\}
$$

For $i \in \mathbb{N}_{N}$, define functions $\omega_{i}(x, y)=\left(L_{i}(x), F_{i}(x, y)\right)$. It is easy to check that each $\omega_{i}$ is a contraction map in $\mathbb{R}$. We need the required IFS $\left\{I \times \mathbb{R} ; \omega_{i}: i \in \mathbb{N}_{N}\right\}$ for construction of a fractal function. The following is the most fundamental result in the field of fractal interpolation.

Theorem 2.1 ([21]). The following hold:

(i) The IFS $\left\{I \times \mathbb{R} ; \omega_{i}: i \in \mathbb{N}_{N}\right\}$ has a unique attractor $G$ such that $G$ is the graph of a continuous function $f: I \rightarrow \mathbb{R}$.

(ii) The function $f$ interpolates the data set, i.e., $f\left(x_{j}\right)=y_{j}$ for all $j$. 
(iii) Let $\mathcal{G}:=\left\{g \in \mathcal{C}(I): g\left(x_{0}\right)=y_{0}, g\left(x_{N}\right)=y_{N}\right\}$ be endowed with the uniform metric $d(g, \tilde{g}):=\max \{|g(x)-\tilde{g}(x)|: x \in I\}$. If $T: \mathcal{G} \rightarrow \mathcal{G}$ is defined by $\operatorname{Tg}(x)=F_{i}\left(L_{i}^{-1}(x), g \circ L_{i}^{-1}(x)\right), x \in I_{i}, i \in \mathbb{N}_{N}$, and for all $g \in \mathcal{G}$, then $T$ has a unique fixed point $f$, and $f=\lim _{n \rightarrow \infty} T^{n}(g)$ for any $g \in \mathcal{G}$. Further, the fixed point $f$ is the function satisfying conditions given in (i)-(ii).

The function which has made its debut in the foregoing theorem is termed a fractal interpolation function (FIF), and it satisfies the functional equation:

$$
f(x)=F_{i}\left(L_{i}^{-1}(x), f \circ L_{i}^{-1}(x)\right), \quad x \in I_{i}, \quad i \in \mathbb{N}_{N} .
$$

The adjective fractal is used to emphasize that the graph of $f$ may have non-integer Hausdorff and Minkowski dimension. Also, the graph $G(f)$ is a union of transformed copies of itself, specifically $G(f)=\bigcup_{i=1}^{N} \omega_{i}(G(f))$. The most extensively studied FIF in theory and applications is formed by

$$
L_{i}(x)=a_{i} x+e_{i}, \quad F_{i}(x, y)=\alpha_{i} y+q_{i}(x), \quad i \in \mathbb{N}_{N},
$$

where $\alpha_{i}$ are constants satisfying $0 \leq\left|\alpha_{i}\right|<1$ and $q_{i}$ are continuous functions so that the "join-up conditions" in (2.2) imposed on the bivariate functions $F_{i}$ are satisfied. The multiplier $\alpha_{i}$ is called a vertical scaling factor for the transformation $\omega_{i}$ and the vector $\alpha=\left(\alpha_{1}, \alpha_{2}, \ldots, \alpha_{N}\right) \in(-1,1)^{N}$ is called a scale vector of the FIF. The prescriptions in (2.1) uniquely determine the constants $a_{i}$ and $b_{i}$ appearing in the affine map $L_{i}$ as

$$
a_{i}=\frac{x_{i}-x_{i-1}}{x_{N}-x_{0}}, \quad e_{i}=\frac{x_{i-1} x_{N}-x_{i} x_{0}}{x_{N}-x_{0}} .
$$

Let us recall that the function $f$ determined by the IFS in (2.4), which takes the form

$$
f(x)=\alpha_{i} f\left(L_{i}^{-1}(x)\right)+q_{i}\left(L_{i}^{-1}(x)\right), \quad x \in I_{i}, i \in \mathbb{N}_{N},
$$

is, in general, non-smooth in nature. The following theorem provides the conditions on $\alpha_{i}$ and functions $q_{i}$ so that the FIF is $\mathcal{C}^{p}$-continuous.

Theorem 2.2 ([2]). Let $\left\{\left(x_{j}, y_{j}\right): j \in \mathbb{N}_{N} \cup\{0\}\right\}$ be a given data set with strictly increasing abscissae. Let $L_{i}(x)=a_{i} x+b_{i}$ satisfy (2.1) and $F_{i}(x, y)=\alpha_{i} y+q_{i}(x)$ obeys (2.2) for $i \in \mathbb{N}_{N}$. Suppose that for some integer $p \geq 0,\left|\alpha_{i}\right|<a_{i}^{p}$ and $q_{i} \in \mathcal{C}^{p}(I), i \in \mathbb{N}_{N}$. Let

$$
F_{i, k}(x, y)=\frac{\alpha_{i} y+q_{i}^{(k)}(x)}{a_{i}^{k}}, \quad y_{0, k}=\frac{q_{1}^{(k)}\left(x_{0}\right)}{a_{1}^{k}-\alpha_{1}}, \quad y_{N, k}=\frac{q_{N}^{(k)}\left(x_{N}\right)}{a_{N}^{k}-\alpha_{N}}, \quad k=1,2, \ldots, p .
$$

If $F_{i-1, k}\left(x_{N}, y_{N, k}\right)=F_{i, k}\left(x_{0}, y_{0, k}\right)$ for $i \in \mathbb{N}_{N}$ and $k \in \mathbb{N}_{p}$, then the IFS $\left\{I \times \mathbb{R} ;\left(L_{i}(x), F_{i}(x, y)\right): i \in \mathbb{N}_{N}\right\}$ determines a FIF $f \in \mathcal{C}^{p}(I)$, and $f^{(k)}$ is the FIF defined by $\left\{I \times \mathbb{R} ;\left(L_{i}(x), F_{i, k}(x, y)\right): i \in \mathbb{N}_{N}\right\}$ for $k \in \mathbb{N}_{p}$.

Based on this theorem, smooth polynomial FIFs are constructed in $[3,6,9]$.

\subsection{A two-point boundary value problem}

Definition 2.1. Let $f: \mathbb{R}^{3} \rightarrow \mathbb{R}$ be a given function. The problem

$$
y^{\prime \prime}=f\left(x, y, y^{\prime}\right), \quad x \in\left[x_{0}, x_{N}\right],
$$

along with the boundary conditions

$$
\left\{\begin{array}{l}
\beta_{1} y\left(x_{0}\right)+\beta_{2} y^{\prime}\left(x_{0}\right)=\beta_{3}, \\
\gamma_{1} y\left(x_{N}\right)+\gamma_{2} y^{\prime}\left(x_{N}\right)=\gamma_{3},
\end{array}\right.
$$

where $\beta_{1}, \beta_{2}, \beta_{3}, \gamma_{1}, \gamma_{2}$ and $\gamma_{3}$ are real numbers, is called a two-point second order boundary value problem (BVP). The existence and uniqueness of the solution of two point BVP have been discussed by Keller [22]. Bickley [10] considered this BVP when (2.6) is taken as

$$
P(x) u^{\prime \prime}+Q(x) u^{\prime}+R(x) u=S(x) .
$$

A BVP (2.6)-(2.7) should be well posed in order to be useful in the applications, which means that there exists a unique solution, which continuously depends on the input imposed to the problem. There are several types of schemes proposed to obtain numerical solutions like shooting method, finite difference schemes, finite element and finite volume methods at discretized points of the domain. When the number of discretized points is very large, the numerical solution is close to the original solution if the concerned method is convergent. Similarly, various types of splines have been used to get the solution of BVP in a closed manner. But this closed forms of the classical solutions does not match with the exact solution, when $R(x)$ is not differentiable in a dense subset of the domain. Thus, we propose a deterministic fractal cubic spline solution for this second order BVP with Dirichlet's boundary conditions, which is more general than the classical cubic spline solution. 


\section{Solution of BVP by fractal splines}

In this section, we solve the two-point BVP by using a cubic spline FIF through moments, which was proposed in [3]. The main idea is to use the condition of continuity of the derivative of the fractal spline at the grid points in the discretization of (2.6) for the computation of moments so that we can write the fractal cubic spline solution explicitly.

\subsection{Cubic spline FIF through moments formulation}

In the first part, we describe the general construction of cubic spline FIFs $f \in \mathcal{C}^{2}\left[x_{0}, x_{N}\right]$ through the moments, $M_{j}=f^{\prime \prime}\left(x_{j}\right), j \in \mathbb{N}_{N} \cup\{0\}$, which passes through the interpolation data set $\left\{\left(x_{j}, y_{j}\right): j \in \mathbb{N}_{N} \cup\{0\}\right\}$ (see [3] for details). Let $\Delta$ represent the partition $x_{0}<x_{1}<\cdots<x_{N}$ of the domain of the interval $I:=\left[x_{0}, x_{N}\right]$ of the BVP. The graph of the cubic spline FIF $f$ is the attractor of the IFS $\mathcal{I}:=\left\{I \times \mathbb{R} ; \omega_{i}(x, y)=\left(L_{i}(x), F_{i}(x, y)\right), i \in \mathbb{N}_{N}\right\}$, where $L_{i}(x)$ satisfies $(2.1)$ and $F_{i}(x, y)=a_{i}^{2}\left(\alpha_{i} y+q_{i}(x)\right),\left|\alpha_{i}\right|<1$, and $q_{i}(x)$ is a suitable cubic polynomial.

Using the moments $M_{j}, j \in \mathbb{N}_{N} \cup\{0\}, f^{\prime}\left(x_{0}\right)$ and $f^{\prime}\left(x_{N}\right)$ in the structure of a $\mathcal{C}^{2}$-fractal cubic spline, we will get $N+1$ equations. In order to compute these $N+3$ unknowns, we have to put two additional boundary conditions similar to the construction of the classical $\mathcal{C}^{2}$-cubic splines. This construction helps us solve a system of order at most $N+3$ equations instead of a system of $4 N$ equations for the computations of the cubic polynomials $q_{i}(x), i \in \mathbb{N}_{N}$. Thus, we will assume the structure of the cubic spline FIF through moments in the following:

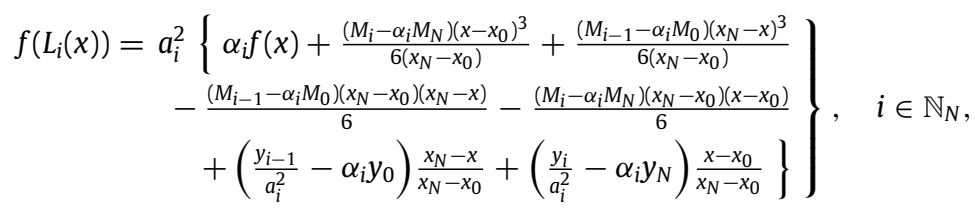

where

$$
L_{i}(x)=a_{i} x+e_{i}, \quad a_{i}=\frac{x_{i}-x_{i-1}}{x_{N}-x_{0}}, \quad \text { and } \quad e_{i}=\frac{x_{N} x_{i-1}-x_{0} x_{i}}{x_{N}-x_{0}} .
$$

Now differentiating (3.1) with respect to $x$, we obtain

$$
\begin{aligned}
f^{\prime}\left(L_{i}(x)\right)= & a_{i}\left\{\alpha_{i} f^{\prime}(x)+\frac{\left(M_{i}-\alpha_{i} M_{N}\right)\left(x-x_{0}\right)^{2}}{2\left(x_{N}-x_{0}\right)}-\frac{\left(M_{i-1}-\alpha_{i} M_{0}\right)\left(x_{N}-x\right)^{2}}{2\left(x_{N}-x_{0}\right)}\right. \\
& +\frac{\left(M_{i-1}-\alpha_{i} M_{0}\right)\left(x_{N}-x_{0}\right)}{6}-\frac{\left(M_{i}-\alpha_{i} M_{N}\right)\left(x_{N}-x_{0}\right)}{6} \\
& \left.-\left(\frac{y_{i-1}}{a_{i}^{2}}-\alpha_{i} y_{0}\right) \frac{1}{x_{N}-x_{0}}+\left(\frac{y_{i}}{a_{i}^{2}}-\alpha_{i} y_{N}\right) \frac{1}{x_{N}-x_{0}}\right\},
\end{aligned}
$$

Let $\ell=x_{N}-x_{0}$. Then, the above equation can be written as

$$
\begin{aligned}
f^{\prime}\left(L_{i}(x)\right)= & a_{i}\left\{\alpha_{i} f^{\prime}(x)+\frac{\left(M_{i}-\alpha_{i} M_{N}\right)\left(x-x_{0}\right)^{2}}{2 \ell}-\frac{\left(M_{i-1}-\alpha_{i} M_{0}\right)\left(x_{N}-x\right)^{2}}{2 \ell}\right. \\
& \left.+\frac{\ell}{6}\left\{M_{i-1}-M_{i}+\alpha_{i}\left(M_{N}-M_{0}\right)\right\}+\frac{1}{\ell}\left(\frac{y_{i}-y_{i-1}}{a_{i}^{2}}-\alpha_{i}\left(y_{N}-y_{0}\right)\right)\right\} .
\end{aligned}
$$

Since $f^{\prime}(x)$ is continuous at the knots $x_{i}, i=1 \ldots, N-1$, we have $\lim _{x \rightarrow x_{i}^{-}} f^{\prime}(x)=\lim _{x \rightarrow x_{i}^{+}} f^{\prime}(x)$ for $i \in \mathbb{N}_{N-1}$. As per assumption (2.1), $L_{i}\left(x_{N}\right)=L_{i+1}\left(x_{0}\right)=x_{i}$. For $d_{N}:=f^{\prime}\left(x_{N}\right)$, substituting $x=x_{N}$ in (3.2), we deduce

$$
\begin{aligned}
f^{\prime}\left(x_{i}^{-}\right)= & a_{i}\left\{\alpha_{i} d_{N}+\frac{\ell}{2}\left(M_{i}-\alpha_{i} M_{N}\right)+\frac{\ell}{6}\left[M_{i-1}-M_{i}+\alpha_{i}\left(M_{N}-M_{0}\right)\right]\right. \\
& \left.+\frac{1}{\ell}\left(\frac{y_{i}-y_{i-1}}{a_{i}^{2}}-\alpha_{i}\left(y_{N}-y_{0}\right)\right)\right\}, \quad i \in \mathbb{N}_{N} .
\end{aligned}
$$

Now, for $d_{0}:=f^{\prime}\left(x_{0}\right)$, putting $x=x_{0}$ in $f^{\prime}\left(L_{i+1}(x)\right)$, we get

$$
\begin{aligned}
f^{\prime}\left(x_{i}^{+}\right)= & a_{i+1}\left\{\alpha_{i+1} d_{0}-\frac{\ell}{2}\left(M_{i}-\alpha_{i+1} M_{0}\right)+\frac{\ell}{6}\left[M_{i}-M_{i+1}+\alpha_{i+1}\left(M_{N}-M_{0}\right)\right]\right. \\
& \left.+\frac{1}{\ell}\left(\frac{y_{i+1}-y_{i}}{a_{i+1}^{2}}-\alpha_{i+1}\left(y_{N}-y_{0}\right)\right)\right\}, \quad i \in \mathbb{N}_{N-1} \cup\{0\} .
\end{aligned}
$$


Denote $h_{i}=x_{i}-x_{i-1}, i \in \mathbb{N}_{N}$. Using (3.3)-(3.4) and continuity at internal grid points, we get the following equations:

$$
\begin{aligned}
& \frac{h_{i}}{6} M_{i-1}+\frac{h_{i}+h_{i+1}}{3} M_{i}+\frac{h_{i+1}}{6} M_{i+1}= \\
& \frac{1}{h_{i}} y_{i-1}-\left(\frac{1}{h_{i}}+\frac{1}{h_{i+1}}\right) y_{i}+\frac{1}{h_{i+1}} y_{i+1}-\left\{a_{i} \alpha_{i} d_{N}-a_{i+1} \alpha_{i+1} d_{0}-\frac{1}{6}\left(2 h_{i} \alpha_{i}\right.\right. \\
& \left.\left.+h_{i+1} \alpha_{i+1}\right) M_{N}-\frac{1}{6}\left(h_{i} \alpha_{i}+2 h_{i+1} \alpha_{i+1}\right) M_{0}+\frac{1}{\ell}\left(a_{i+1} \alpha_{i+1}-a_{i} \alpha_{i}\right)\left(y_{N}-y_{0}\right)\right\} \\
& \text { for } i=1,2, \ldots, N-1 .
\end{aligned}
$$

Substituting $i=1$ and $x=x_{0}$ in (3.2), the functional relation for $d_{0}$ is

$$
\begin{aligned}
d_{0}= & a_{1} \alpha_{1} d_{0}-\frac{h_{1}}{2}\left(1-\alpha_{1}\right) M_{0}+\frac{h_{1}}{6}\left(\left(1-\alpha_{1}\right) M_{0}-M_{1}+\alpha_{1} M_{N}\right) \\
& +\frac{a_{1}}{\ell}\left(\frac{y_{1}-y_{0}}{a_{1}^{2}}-\alpha_{1}\left(y_{N}-y_{0}\right)\right) \\
\Rightarrow\left(1-a_{1} \alpha_{1}\right) d_{0}= & \frac{y_{1}-y_{0}}{h_{1}}-\frac{a_{1} \alpha_{1}}{\ell}\left(y_{N}-y_{0}\right)-\frac{h_{1}}{3}\left(1-\alpha_{1}\right) M_{0} \\
& -\frac{h_{1}}{6}\left(M_{1}-\alpha_{1} M_{N}\right) .
\end{aligned}
$$

Similarly, substituting $i=N$ and $x=x_{N}$ in (3.2), the functional relation for $d_{N}$ is

$$
\begin{aligned}
d_{N}= & a_{N} \alpha_{N} d_{N}+\frac{h_{N}}{2}\left(\left(1-\alpha_{N}\right) M_{N}\right)+\frac{h_{N}}{6}\left[M_{N-1}-M_{N}+\alpha_{N}\left(M_{N}\right.\right. \\
& \left.\left.-M_{0}\right)\right]+\frac{a_{N}}{\ell}\left(\frac{y_{N}-y_{N-1}}{a_{N}^{2}}-\alpha_{N}\left(y_{N}-y_{0}\right)\right), \\
\Rightarrow\left(1-a_{N} \alpha_{N}\right) d_{N}= & \frac{y_{N}-y_{N-1}}{h_{N}}-\frac{a_{N} \alpha_{N}}{\ell}\left(y_{N}-y_{0}\right)+\frac{h_{N}}{3}\left(1-\alpha_{N}\right) M_{N} \\
& +\frac{h_{N}}{6}\left(M_{N-1}-\alpha_{N} M_{0}\right) .
\end{aligned}
$$

Thus, we have $N+1$ equations in (3.5)-(3.7) for the computation of IFS parameters $M_{j}, j \in \mathbb{N}_{N} \cup\{0\}, d_{0}=f^{\prime}\left(x_{0}\right)$ and $d_{N}=f^{\prime}\left(x_{N}\right)$. Our aim is to replace these $M_{j}$ with functional values at grid points with suitable discretization of the given differential equation.

\subsection{Discretization of BVP by fractal splines}

Now we wish to solve a linear two-point second order BVP of the form

$$
y^{\prime \prime}(x)+P(x) y^{\prime}(x)+Q(x) y(x)=R(x)
$$

with Dirichlet boundary conditions

$$
y\left(x_{0}\right)=\beta^{*} \quad \text { and } \quad y\left(x_{N}\right)=\gamma^{*},
$$

where $R(x)$ may be continuous and nowhere differentiable function in $I:=\left[x_{0}, x_{N}\right]$. If we use any classical spline solution $\psi$ of the BVP (3.8)-(3.9), then $\psi^{\prime \prime}(x)+P(x) \psi^{\prime}(x)+Q(x) \psi(x)$ is a piecewise differentiable function which does not match with $R(x)$. Therefore, we need a $\mathcal{C}^{2}$-function whose 2 nd derivative may be similar to a continuous and nowhere differentiable function. This can be easily achieved by a fractal cubic spline. First, we will discretize the domain $\left[x_{0}, x_{N}\right]$ as $x_{0}<x_{1}<x_{2}<\cdots<x_{N-1}<x_{N}$. We have to find the approximate values of the solution as $y_{j}$ at the grid $x_{j}$ for $j=1,2, \ldots, N-1$ through the fractal cubic spline solution (3.1). We compute the approximate solution of the BVP using (3.3)-(3.7) in our methodology.

At $x=x_{i}$, (3.8) can be written in the discretization form as

$$
M_{i}+P_{i} d_{i}+Q_{i} y_{i}=R_{i} \text { for } i=0,1,2, \ldots, N,
$$

where $M_{i}=y^{\prime \prime}\left(x_{i}\right), P_{i}=P\left(x_{i}\right), d_{i}=y^{\prime}\left(x_{i}\right), Q_{i}=Q\left(x_{i}\right), y_{i}=y\left(x_{i}\right)$ and $R_{i}=R\left(x_{i}\right)$. Using (3.10) in (3.4), we obtain the following equations for $i=0,1, \ldots, N-1$ :

$$
\left(1-\frac{h_{i+1}}{3} P_{i}\right) M_{i}-\frac{h_{i+1} P_{i}}{6} M_{i+1}=R_{i}+\left(\frac{P_{i}}{h_{i+1}}-Q_{i}\right) y_{i}-\frac{P_{i}}{h_{i+1}} y_{i+1}+K_{0, N}^{1} a_{i+1} \alpha_{i+1} P_{i},
$$

where $K_{0, N}^{1}=-\left[d_{0}+\frac{\ell}{6}\left(M_{N}-M_{0}\right)+\frac{\ell}{2} M_{0}-\frac{1}{\ell}\left(y_{N}-y_{0}\right)\right]$. 
Similarly, using (3.10) in (3.3), we obtain the following equations for $i=1, \ldots, N$ :

$$
\frac{h_{i} P_{i}}{6} M_{i-1}+\left(1+\frac{h_{i}}{3} P_{i}\right) M_{i}=R_{i}-\left(\frac{P_{i}}{h_{i}}+Q_{i}\right) y_{i}+\frac{P_{i}}{h_{i}} y_{i-1}+K_{0, N}^{2} a_{i} \alpha_{i} P_{i}
$$

where $K_{0, N}^{2}=-\left[d_{N}+\frac{\ell}{6}\left(M_{N}-M_{0}\right)-\frac{\ell}{2} M_{N}-\frac{1}{\ell}\left(y_{N}-y_{0}\right)\right]$.

It is clear that (3.11) and (3.12) constitute a system of $2 N$ equations with the $2 N+4$ unknowns, $M_{0}, M_{1}, \ldots, M_{N}$ and $y_{0}, y_{1}, \ldots, y_{N}$ and $d_{0}, d_{N}$. Elimination of $M_{j}$ 's leads directly to $N+1$ unknowns $y_{1}, y_{2}, \ldots, y_{N-1}$ and $d_{0}, d_{N}$, The continuity conditions at the internal grid points along with the two boundary conditions are sufficient for their determination. Addition of (3.11) and (3.12) yields the following relations for $i=1,2, \ldots, N-1$ :

$$
\begin{aligned}
\frac{h_{i}}{6} P_{i} M_{i-1} & +\left[2-\frac{h_{i+1}-h_{i}}{3}\right] M_{i}-\frac{h_{i+1}}{6} P_{i} M_{i+1}=2 R_{i}+\frac{P_{i}}{h_{i}} y_{i-1}-\frac{P_{i}}{h_{i+1}} y_{i+1} \\
& -\left[\frac{h_{i+1}-h_{i}}{h_{i} h_{i+1}}+2 Q_{i}\right] y_{i}+\left(K_{0, N}^{1} a_{i+1} \alpha_{i+1}+K_{0, N}^{2} a_{i} \alpha_{i}\right) P_{i} .
\end{aligned}
$$

Elimination of $M_{i}$ from (3.13) and (3.5), leads us to the following equations:

$$
A_{1, i} M_{i-1}+A_{2, i} M_{i+1}=A_{3, i} y_{i-1}+A_{4, i} y_{i}+A_{5, i} y_{i+1}-A_{6, n},
$$

where

$$
\begin{aligned}
A_{1, i}= & \frac{h_{i}}{3}\left(1-\frac{h_{i+1} P_{i}}{3}\right), \quad A_{2, i}=\frac{h_{i+1}}{3}\left(1+\frac{h_{i} P_{i}}{3}\right), \\
A_{3, i}= & \frac{2}{h_{i}}\left(1-\frac{h_{i+1} P_{i}}{3}\right), \quad A_{5, i}=\frac{2}{h_{i+1}}\left(1+\frac{h_{i} P_{i}}{3}\right), \\
A_{4, i}= & -2\left\{\left(h_{i}+h_{i+1}\right)\left[\frac{1}{h_{i} h_{i+1}}-\frac{Q_{i}}{3}\right]+\frac{h_{i}^{2}-h_{i+1}^{2}}{3 h_{i} h_{i+1}} P_{i}\right\}, \\
A_{6, i}= & \frac{h_{i}+h_{i+1}}{3}\left[\left(K_{0, N}^{1} a_{i+1} \alpha_{i+1}+K_{0, N}^{2} a_{i} \alpha_{i}\right) P_{i}+2 R_{i}\right]+\left(2+\frac{h_{i}-h_{i+1}}{3} P_{i}\right) K_{i, 0, N}^{1}, \\
K_{i, 0, N}^{1}= & a_{i} \alpha_{i} d_{N}-a_{i+1} \alpha_{i+1} d_{0}-\frac{1}{6}\left(2 h_{i} \alpha_{i}+h_{i+1} \alpha_{i+1}\right) M_{N} \\
& -\frac{1}{6}\left(h_{i} \alpha_{i}+2 h_{i+1} \alpha_{i+1}\right) M_{0}+\frac{1}{\ell}\left(a_{i+1} \alpha_{i+1}-a_{i} \alpha_{i}\right)\left(y_{N}-y_{0}\right) .
\end{aligned}
$$

But an explicit form of $M_{i-1}$ can be obtained in terms of $y_{i-1}$ and $y_{i}$ by eliminating $M_{i}$ from (3.11) (by replacing $i$ by $i-1$ ) and (3.12) as

$$
A_{7, i} M_{i-1}=A_{8, i} y_{i-1}-A_{9, i} y_{i}+A_{10, i}
$$

where

$$
\begin{aligned}
& A_{7, i}=\left(1-\frac{h_{i}}{3} P_{i-1}\right)\left(1+\frac{h_{i}}{3} P_{i}\right)+\frac{h_{i}^{2}}{36} P_{i-1} P_{i}, \\
& A_{8, i}=\left(1+\frac{h_{i}}{3} P_{i}\right)\left(\frac{P_{i-1}}{h_{i}}-Q_{i-1}\right)+\frac{P_{i} P_{i-1}}{6}, \\
& A_{9, i}=\left(1+\frac{h_{i}}{3} P_{i}\right) \frac{P_{i-1}}{h_{i}}+\frac{h_{i} P_{i-1}}{6}\left(\frac{P_{i}}{h_{i}}+Q_{i}\right), \\
& A_{10, i}=\left[\left(1+\frac{h_{i} P_{i}}{3}\right) K_{0, N}^{1}+\frac{h_{i}}{6} K_{0, N}^{2} P_{i}\right] a_{i} \alpha_{i} P_{i-1}+\left(1+\frac{h_{i}}{3} P_{i}\right) R_{i-1}+\frac{h_{i} P_{i-1}}{6} R_{i} .
\end{aligned}
$$

Similarly, an explicit form of $M_{i+1}$ can be obtained in terms of $y_{i}$ and $y_{i+1}$ by eliminating $M_{i}$ from (3.11) (by replacing $i$ by $i+1)$ and (3.12) as

$$
A_{11, i} M_{i+1}=A_{12, i} y_{i}+A_{13, i} y_{i+1}+A_{14, i},
$$


where

$$
\begin{aligned}
A_{11, i}= & \left(1-\frac{h_{i+1}}{3} P_{i}\right)\left(1+\frac{h_{i+1}}{3} P_{i+1}\right)+\frac{h_{i+1}^{2}}{36} P_{i} P_{i+1}, \\
A_{12, i}= & \left(1-\frac{h_{i+1}}{3} P_{i}\right) \frac{P_{i+1}}{h_{i+1}}-\frac{h_{i+1} P_{i+1}}{6}\left(\frac{P_{i}}{h_{i+1}}-Q_{i+1}\right), \\
A_{13, i}= & \frac{P_{i} P_{i+1}}{6}-\left(1-\frac{h_{i+1}}{3} P_{i}\right)\left(\frac{P_{i+1}}{h_{i+1}}-Q_{i+1}\right), \\
A_{14, i}= & {\left[\left(1-\frac{h_{i+1} P_{i}}{3}\right) K_{0, N}^{1}-\frac{h_{i+1}}{6} K_{0, N}^{2} P_{i}\right] a_{i+1} \alpha_{i+1} P_{i+1}+\left(1-\frac{h_{i+1}}{3} P_{i}\right) R_{i+1} } \\
& +\frac{h_{i+1} P_{i+1}}{6} R_{i} .
\end{aligned}
$$

Substituting $M_{i-1}$ and $M_{i+1}$ in (3.14), we obtain the following equations in terms of $y_{i-1}, y_{i}$ and $y_{i+1}$ :

$$
\Lambda_{1, i} y_{i+1}+\Lambda_{2, i} y_{i}+\Lambda_{3, i} y_{i-1}=\Lambda_{4, i} R_{i+1}+\Lambda_{5, i} R_{i}+\Lambda_{6, i} R_{i-1}+\Lambda_{7, i}, \quad i \in \mathbb{N}_{N-1},
$$

where

$$
\begin{aligned}
\Lambda_{1, i}= & \left(1-\frac{h_{i+1}}{3} P_{i}\right)\left(1+\frac{h_{i}}{3} P_{i}\right)\left[P_{i-1}-\frac{h_{i}}{3} Q_{i-1}-\frac{2}{h_{i}}\right] A_{11, i}, \\
\Lambda_{2, i}= & \left(1-\frac{h_{i+1}}{3} P_{i}\right)\left(1+\frac{h_{i}}{3} P_{i}\right)\left[\frac{1}{h_{n+1}}\left(1+\frac{h_{i+1} P_{i+1}}{2}\right) A_{7, i}+\frac{1}{h_{i}}\left(1-\frac{h_{i-1} P_{i-1}}{2} A_{11, i}\right)\right. \\
& \left.-\frac{1}{3} C_{i} Q_{i}\right], \\
\Lambda_{3, i}= & -\left(1-\frac{h_{i+1}}{3} P_{i}\right)\left(1+\frac{h_{i}}{3} P_{i}\right)\left[P_{i+1}+\frac{h_{i+1}}{3} Q_{i+1}+\frac{2}{h_{i+1}}\right] A_{7, i}, \\
\Lambda_{4, i}= & -\frac{h_{i}}{3}\left(1-\frac{h_{i+1}}{3} P_{i}\right)\left(1+\frac{h_{i}}{3} P_{i}\right) A_{11, i}, \\
\Lambda_{5, i}= & \frac{h_{i+1}^{2} P_{i+1}}{18}\left(1+\frac{h_{i} P_{i}}{3}\right) A_{7, i}-\frac{h_{i}^{2} P_{i-1}}{18}\left(1-\frac{h_{i+1} P(i)}{3}\right) A_{11, i}, \\
\Lambda_{6, i}= & -\frac{h_{i+1}}{3}\left(1-\frac{h_{i+1}}{3} P_{i}\right)\left(1+\frac{h_{i}}{3} P_{i}\right) A_{7, i}, \\
\Lambda_{7, i}= & {\left[K_{0, N}^{1} a_{i+1} \alpha_{i+1}+K_{0, N}^{2} a_{i} \alpha_{i}+\left(2-\frac{h_{i+1}-h_{i}}{3} P_{i}\right) K_{i, 0, N}^{1}\right] A_{7, i} A_{11, i} } \\
& -\frac{h_{i}}{3}\left(1-\frac{h_{i+1}}{3} P_{i}\right)\left[\left(1+\frac{h_{i}}{3} P_{i}\right) K_{0, N}^{1}+\frac{h_{i}}{6} K_{0, N}^{2} P_{i}\right] a_{i} \alpha_{i} P_{i-1} A_{11, i} \\
& -\frac{h_{i+1}}{3}\left(1+\frac{h_{i}}{3} P_{i}\right)\left[\left(1-\frac{h_{i+1}}{3} P_{i}\right) K_{0, N}^{1}-\frac{h_{i+1}}{6} K_{0, N}^{2} P_{i}\right] a_{i+1} \alpha_{i+1} P_{i+1} A_{7, i} \\
C_{i}= & \left(h_{i}+h_{i+1}\right)-\frac{3 h_{i}+4 h_{i+1}}{12} h_{i} P_{i-1}+\frac{3 h_{i+1}+4 h_{i}}{12} h(i+1) P_{i+1} \\
& \frac{h_{i} h_{i+1}}{12}\left(h_{i}+h_{i+1}\right) P_{i-1} P_{i+1} .
\end{aligned}
$$

Solving (3.17) in conjunction with the boundary conditions in (3.9), we compute the values of $y_{j}\left(j \in \mathbb{N}_{N} \cup\{0\}\right)$. Using (3.15) and (3.16), we will get the values of all moments $M_{i}, i \in \mathbb{N}_{N} \cup\{0\}$. Finally, the cubic spline fractal solution to the BVP is obtained from (3.1).

Remark 3.1. When the scaling factors are taken zeros and the partition points are equally spaced then the spline FIF (3.1) reduces to the classical spline function as discussed in Albasiny et al. [11].

Remark 3.2. When we select a uniform partition $\left(h_{i}=h\right.$ gives $\left.a_{i}=a=\frac{1}{N}\right)$ and equal scaling factors $\alpha_{i}=\alpha, i \in \mathbb{N}_{N}$, then (3.17) reduces to the following recurrence relation:

$$
\begin{aligned}
\frac{a}{a^{2} h}\left(y_{i-1}-2 y_{i}+y_{i+1}\right) & -\frac{h}{6}\left(P_{i-1} y_{i-1}+4 P_{i} y_{i}+P_{i+1} y_{i+1}\right) \\
& =\frac{h}{6}\left(R_{i-1}+4 R_{i}+R_{i+1}\right)+K_{0}, \quad i \in \mathbb{N}_{N-1} .
\end{aligned}
$$

This clearly corresponds to the central finite-difference representation:

$$
\delta^{2} y_{i}+a^{2} h^{2}\left(1+\frac{\delta^{2}}{6}\right) P_{i} y_{i}=a^{2} h^{2}\left(1+\frac{\delta^{2}}{6}\right) R_{i}+K_{0}, \quad i \in \mathbb{N}_{N-1},
$$


where $K_{0}=a^{2} h \alpha\left(d_{N}-d_{0}\right)+\frac{a h \alpha}{2}\left(P_{0} y_{0}+P_{N} y_{N}\right)-\frac{a h \alpha}{2}\left(R_{0}+R_{N}\right)$, and the central difference is defined as $\delta y_{i}=f\left(y_{i}+\frac{h}{2}\right)-f\left(y_{i}-\frac{h}{2}\right)$. Similarly considering $P(x)$ as a constant $P$ (say), then it is clear that $A_{7, i}=A_{11, i}=C_{i}=\frac{a^{2} h^{2} P^{2}}{12}$ (a cancellation term throughout (3.13)) and (3.13) reduces to the following finite-difference representation:

$$
\delta^{2} y_{i}+\frac{a h P}{2}\left(y_{i+1}-y_{i-1}\right)+a^{2} h^{2}\left(1+\frac{\delta^{2}}{6}\right) Q_{i} y_{i}=a^{2} h^{2}\left(1+\frac{\delta^{2}}{6}\right) R_{i}+K_{i, 0, N}^{2}, \quad i \in \mathbb{N}_{N-1} .
$$

By solving the above algebraic equations, the unknowns $y_{j}, j \in \mathbb{N}_{N} \cup\{0\}$, can be computed. Finally, using these $y_{j}$ and $M_{j}$, we get the analytical fractal solution of BVP from (3.1).

Remark 3.3. When the first derivative is absent i.e., $Q(x)=0$, one can use the above procedure with $Q_{j}=0$ for all $j \in \mathbb{N}_{N} \cup\{0\}$. Otherwise, one can use a simpler way to calculate the moments as described in the following:

In this case, (3.8) reduces to

$$
y^{\prime \prime}(x)+P(x) y(x)=R(x) .
$$

Discretizing (3.18), we get

$$
M_{i}=R_{i}-P_{i} y_{i} \quad \text { for } i=0,1,2, \ldots, N,
$$

where $M_{i}=y^{\prime \prime}\left(x_{i}\right), R_{i}=R\left(x_{i}\right), P_{i}=P\left(x_{i}\right), y_{i}=y\left(x_{i}\right)$ for $i=0,1,2, \ldots, N$.

Using (3.19) in (3.5), we have

$$
\begin{aligned}
& \frac{6+h_{i}^{2} P_{i-1}}{6 h_{i}} y_{i-1}+\left[\frac{P_{i}}{3}-\frac{1}{h_{i} h_{i+1}}\right]\left(h_{i}+h_{i+1}\right) y_{i}+\frac{6+h_{i+1}^{2} P_{i+1}}{6 h_{i+1}} y_{i+1}=\frac{h_{i}+h_{i+1}}{3} R_{i} \\
& \frac{h_{i}}{6} R_{i-1}+\frac{h_{i+1}}{6} R_{i+1}+\left\{a_{i} \alpha_{i} d_{N}-a_{i+1} \alpha_{i+1} d_{0}-\frac{1}{6}\left(2 h_{i} \alpha_{i}+h_{i+1} \alpha_{i+1}\right)\left(R_{N}-P_{N} y_{N}\right)\right. \\
& \left.\quad-\frac{1}{6}\left(h_{i} \alpha_{i}+2 h_{i+1} \alpha_{i+1}\right)\left(R_{0}-P_{0} y_{0}\right)+\frac{1}{\ell}\left(a_{i+1} \alpha_{i+1}-a_{i} \alpha_{i}\right)\left(y_{N}-y_{0}\right)\right\}, \quad i \in \mathbb{N}_{N-1} .
\end{aligned}
$$

From (3.6) and (3.19), we obtain

$$
\begin{gathered}
\left(\frac{1}{h_{1}}-\frac{a_{1} \alpha_{1}}{\ell}+\frac{h_{1}\left(1-\alpha_{1}\right) P_{0}}{3}\right) y_{0}-\frac{6+h_{1}^{2} P_{1}}{6 h_{1}} y_{1}+\frac{6 a_{1}+\ell h_{1} P_{N}}{6 \ell} \alpha_{1} y_{N} \\
=\frac{h_{1}}{3}\left(1-\alpha_{1}\right) R_{0}-\frac{h_{1}}{6}\left(R_{1}-\alpha_{1} R_{N}\right)-\left(1-a_{1} \alpha_{1}\right) d_{0} .
\end{gathered}
$$

Similarly, from (3.7) and (3.19), we get

$$
\begin{gathered}
\frac{6 a_{N}-\ell h_{N} P_{N}}{6 \ell} \alpha_{N} y_{0}-\frac{6-h_{N}^{2} P_{N-1}}{6 h_{N}} y_{N-1}+\left(\frac{1}{h_{N}}-\frac{a_{N} \alpha_{N}}{\ell}+\frac{h_{N}\left(1-\alpha_{N}\right) P_{N}}{3}\right) y_{N} \\
=\frac{h_{N}}{3}\left(1-\alpha_{N}\right) R_{N}+\frac{h_{N}}{6}\left(R_{N-1}-\alpha_{N} R_{0}\right)+\left(1-a_{N} \alpha_{N}\right) d_{N} .
\end{gathered}
$$

Since $y_{0}=y\left(x_{0}\right)=\beta^{*}$ and $y_{N}=y\left(x_{N}\right)=\gamma^{*}$ are available, we need to compute $(N+1)$ unknowns $y_{1}, \ldots, y_{N-1}, d_{0}$ and $d_{N}$ from the system (3.20)-(3.22). Once this system is solved, using the values with $M_{0}, \ldots, M_{N}$ in (3.1), we obtain the desired spline FIF solution to the BVP.

\section{Error analysis}

In this section, we investigate the truncation error corresponding to the proposed method. Assume that the partition $\triangle:=x_{0}<x_{1}<x_{2}<\cdots<x_{N}$ of the domain interval $I:=\left[x_{0}, x_{N}\right]$ is equally spaced. That is $h_{i}=x_{i}-x_{i-1}=h \forall i \in \mathbb{N}_{N}$, where $h=\frac{x_{N}-x_{0}}{N}$, and $a_{i}=a==\frac{1}{N} \forall i \in \mathbb{N}_{N}$. Then (3.5)-(3.7) can be written as:

$$
\begin{aligned}
\frac{h}{6} M_{i-1}+\frac{2 h}{3} M_{i}+\frac{h}{6} M_{i+1}= & \frac{1}{h} y_{i-1}-\left(\frac{1}{h}+\frac{1}{h}\right) y_{i}+\frac{1}{h} y_{i+1}-\left\{a \alpha_{i} d_{N}-a \alpha_{i+1} d_{0}-\frac{1}{6}\left(2 h \alpha_{i}\right.\right. \\
& \left.\left.\quad+h \alpha_{i+1}\right) M_{N}-\frac{1}{6}\left(h \alpha_{i}+2 h \alpha_{i+1}\right) M_{0}+\frac{1}{\ell}\left(a \alpha_{i+1}-a \alpha_{i}\right)\left(y_{N}-y_{0}\right)\right\} \\
\quad \text { for } i=1,2, \ldots, N-1 . & \\
d_{0}= & a \alpha_{1} d_{0}-\frac{h}{2}\left(1-\alpha_{1}\right) M_{0}+\frac{h}{6}\left(\left(1-\alpha_{1}\right) M_{0}-M_{1}+\alpha_{1} M_{N}\right) \\
& +\frac{a}{\ell}\left(\frac{y_{1}-y_{0}}{a^{2}}-\alpha_{1}\left(y_{N}-y_{0}\right)\right),
\end{aligned}
$$




$$
\begin{aligned}
\Rightarrow\left(1-a \alpha_{1}\right) d_{0}= & \frac{y_{1}-y_{0}}{h}-\frac{a \alpha_{1}}{\ell}\left(y_{N}-y_{0}\right)-\frac{h}{3}\left(1-\alpha_{1}\right) M_{0} \\
& -\frac{h}{6}\left(M_{1}-\alpha_{1} M_{N}\right) .
\end{aligned}
$$

and

$$
\begin{aligned}
d_{N}= & a \alpha_{N} d_{N}+\frac{h}{2}\left(\left(1-\alpha_{N}\right) M_{N}\right)+\frac{h}{6}\left[M_{N-1}-M_{N}+\alpha_{N}\left(M_{N}\right.\right. \\
& \left.\left.-M_{0}\right)\right]+\frac{a}{\ell}\left(\frac{y_{N}-y_{N-1}}{a^{2}}-\alpha_{N}\left(y_{N}-y_{0}\right)\right), \\
\Rightarrow\left(1-a \alpha_{N}\right) d_{N}= & \frac{y_{N}-y_{N-1}}{h}-\frac{a \alpha_{N}}{\ell}\left(y_{N}-y_{0}\right)+\frac{h}{3}\left(1-\alpha_{N}\right) M_{N} \\
& +\frac{h}{6}\left(M_{N-1}-\alpha_{N} M_{0}\right) .
\end{aligned}
$$

Assume that $y \in \mathcal{C}^{4}\left[x_{0}, x_{N}\right]$. Let us consider the Taylor series expansion of $y\left(x_{i}\right)$ and $y\left(x_{i+1}\right)$ around the point $x_{i-1}$ as

$$
y\left(x_{i}\right)=y\left(x_{i-1}\right)+h y^{\prime}\left(x_{i-1}\right)+\frac{h^{2}}{2 !} y^{\prime \prime}\left(x_{i-1}\right)+\frac{h^{3}}{3 !} y^{\prime \prime \prime}\left(x_{i-1}\right)+\mathcal{O}\left(h^{4}\right),
$$

and

$$
y\left(x_{i+1}\right)=y\left(x_{i-1}\right)+2 h y^{\prime}\left(x_{i-1}\right)+2 h^{2} y^{\prime \prime}\left(x_{i-1}\right)+\frac{4 h^{3}}{3} y^{\prime \prime \prime}\left(x_{i-1}\right)+\mathcal{O}\left(h^{4}\right) .
$$

It is clear that

$$
\frac{-y\left(x_{i+1}\right)+4 y\left(x_{i}\right)-3 y\left(x_{i-1}\right)}{2 h}=y^{\prime}\left(x_{i-1}\right)-\frac{h^{2}}{3} y^{\prime \prime \prime}\left(x_{i-1}\right)+\mathcal{O}\left(h^{3}\right) .
$$

Using the Taylor series expansion of $y\left(x_{i-1}\right)$ and $y\left(x_{i+1}\right)$ around the point $x_{i}$, we obtain the following:

$$
\frac{y\left(x_{i+1}\right)-y\left(x_{i-1}\right)}{2 h}=y^{\prime}\left(x_{i}\right)+\frac{h^{2}}{3 !} y^{\prime \prime \prime}\left(x_{i}\right)+\mathcal{O}\left(h^{4}\right) \text {. }
$$

Similarly using the Taylor series expansion of $y\left(x_{i-1}\right)$ and $y\left(x_{i}\right)$ around the point $x_{i+1}$, we obtain the following:

$$
\frac{3 y\left(x_{i+1}\right)-4 y\left(x_{i}\right)+y\left(x_{i-1}\right)}{2 h}=y^{\prime}\left(x_{i+1}\right)-\frac{h^{2}}{3} y^{\prime \prime \prime}\left(x_{i+1}\right)+\mathcal{O}\left(h^{3}\right) \text {. }
$$

Hence, the following second order approximations to $d_{i}$ can be used.

$$
d_{i}=\frac{y_{i+1}-y_{i-1}}{2 h}, \quad d_{i+1}=\frac{3 y_{i+1}-4 y_{i}+y_{i-1}}{2 h}, \quad d_{i-1}=\frac{-y_{i+1}+4 y_{i}-3 y_{i-1}}{2 h} .
$$

Substituting $M_{i}=R_{i}-P_{i} d_{i}-Q_{i} y_{i}$ and $d_{i}$ for $i=0,1, N$ in (4.1), we get

$$
\begin{aligned}
& {\left[-3 h\left(1-a \alpha_{1}\right)+h^{2}\left(1-\alpha_{1}\right) P_{0}+\frac{h^{2} P_{1}}{6}+2 h-\frac{2 h^{3} \alpha_{1}}{\ell^{2}}-\frac{2 h^{3}\left(1-\alpha_{1}\right) Q_{0}}{3}\right] y_{0}+\left[4 h\left(1-a \alpha_{1}\right)\right.} \\
& \left.-\frac{4 h^{2}\left(1-\alpha_{1}\right) P_{0}}{3}-2 h-\frac{h^{3} Q_{1}}{3}\right] y_{1}+\left[-h\left(1-a \alpha_{1}\right)+\frac{h^{2}\left(1-\alpha_{1}\right) P_{0}}{3}-\frac{h^{2} P_{1}}{6}\right] y_{2}+\frac{h^{2} \alpha_{1} P_{N}}{6} y_{N-2} \\
& -\frac{2 h^{2} \alpha_{1} P_{N}}{3} y_{N-1}+h^{2} \alpha_{1}\left[\frac{P_{N}}{2}+\frac{2 h \alpha_{1}}{\ell^{2}}+\frac{h \alpha_{1} Q_{N}}{3}\right] y_{N}=-\frac{2 h^{3}\left(1-\alpha_{1}\right)}{3} R_{0}-\frac{h^{3}}{3} R_{1}+\frac{h^{3} \alpha_{1}}{3} R_{N} .
\end{aligned}
$$

Replacing $M_{i}=R_{i}-P_{i} d_{i}-Q_{i} y_{i}$ and $d_{i}$ for $i=0, N-1, N$ in (4.2), we deduce

$$
\begin{aligned}
& {\left[3 h\left(1-a \alpha_{N}\right)+h^{2}\left(1-\alpha_{N}\right) P_{N}+\frac{h^{2} P_{N-1}}{6}-2 h+\frac{2 h^{3} \alpha_{N}}{\ell^{2}}-\frac{2 h^{3}\left(1-\alpha_{N}\right) Q_{N}}{3}\right] y_{N}+\left[-4 h\left(1-a \alpha_{N}\right)\right.} \\
& \left.-\frac{4 h^{2}\left(1-\alpha_{N}\right) P_{N}}{3}+2 h+\frac{h^{3} Q_{N-1}}{3}\right] y_{N-1}+\left[h\left(1-a \alpha_{N}\right)+\frac{h^{2}\left(1-\alpha_{N}\right) P_{N}}{3}-\frac{h^{2} P_{N-1}}{6}+2 h+\frac{h^{3} Q_{N-1}}{3}\right] y_{N-2} \\
& -\frac{h^{2} \alpha_{N} P_{0}}{6} y_{2}+\frac{2 h^{2} \alpha_{N} P_{0}}{3} y_{1}-h^{2} \alpha_{N}\left[\frac{P_{0}}{2}+\frac{2 h \alpha_{N}}{\ell^{2}}+\frac{h \alpha_{N} Q_{0}}{3}\right] y_{0}=\frac{2 h^{3}\left(1-\alpha_{N}\right)}{3} R_{N}+\frac{h^{3}}{3} R_{N-1}-\frac{h^{3} \alpha_{N}}{3} R_{0} .
\end{aligned}
$$

Similarly, substituting $M_{j}=R_{j}-P_{j} d_{j}-Q_{j} y_{j}$ and $d_{j}$ for $j=0, N, i-1, i, i+1$ in (4.3), we have

$$
\begin{aligned}
& {\left[6+h^{2} Q_{i-1}-\frac{3 h P_{i-1}}{2}-2 h P_{i}+\frac{h P_{i+1}}{2}\right] y_{i-1}+\left[4 h^{2} Q_{i}-12+2 h P_{i-1}-2 h P_{i}-2 h P_{i+1}\right] y_{i}} \\
& {\left[6+h_{i+1}^{Q}-\frac{h P_{i-1}}{2}+2 h P_{i}+\frac{3 h P_{i+1}}{2}\right] y_{i+1}+\left[h^{2}\left(\alpha_{i}+2 \alpha_{i+1} Q_{0}-\frac{6 h^{2}\left(\alpha_{i+1}-\alpha_{i}\right)}{\ell^{2}}\right)-\frac{9 h \alpha_{i+1}}{\ell}\right.}
\end{aligned}
$$




$$
\begin{aligned}
& \left.+\frac{3 h\left(\alpha_{i}+2 \alpha_{i+1}\right) P_{0}}{2}\right] y_{0}+\left[\frac{12 h \alpha_{i+1}}{\ell}+2 h\left(\alpha_{i}+2 \alpha_{i+1}\right) P_{0}\right] y_{1}-\left[\frac{3 h \alpha_{i+1}}{\ell}+\frac{h\left(\alpha_{i}+2 \alpha_{i+1}\right)}{2} P_{0}\right] y_{2} \\
& -\left[\frac{3 h \alpha_{i}}{\ell}+\frac{h\left(2 \alpha_{i}+\alpha_{i+1}\right)}{2} P_{N}\right] y_{N-2}+\left[\frac{12 h \alpha_{i}}{\ell}+2 h\left(2 \alpha_{i}+\alpha_{i+1}\right) P_{N}\right] y_{N-1}-\left[\frac{9 h \alpha_{i}}{\ell}+\frac{3 h\left(2 \alpha_{i}+\alpha_{i+1}\right) P_{N}}{2}\right] \\
& =h^{2} R_{i-1}+4 h^{2} R_{i}+h^{2} R_{i+1}+h^{2}\left(\alpha_{i}+2 \alpha_{i+1}\right) R_{0}-h^{2}\left(2 \alpha_{i}+\alpha_{i+1}\right) R_{N} .
\end{aligned}
$$

Now let $\left|\alpha_{i}\right|<a^{2}=\frac{h^{2}}{\ell^{2}}$. We get the truncation error $T_{0}(h)$ associated with the equation given in (4.4) as

$$
\begin{aligned}
T_{0}(h)= & {\left[-3 h\left(1-a \alpha_{1}\right)+h^{2}\left(1-\alpha_{1}\right) P_{0}+\frac{h^{2} P_{1}}{6}+2 h-\frac{2 h^{3} \alpha_{1}}{\ell^{2}}-\frac{2 h^{3}\left(1-\alpha_{1}\right) Q_{0}}{3}\right] y_{0}+\left[4 h\left(1-a \alpha_{1}\right)\right.} \\
& \left.-\frac{4 h^{2}\left(1-\alpha_{1}\right) P_{0}}{3}-2 h-\frac{h^{3} Q_{1}}{3}\right] y_{1}+\left[-h\left(1-a \alpha_{1}\right)+\frac{h^{2}\left(1-\alpha_{1}\right) P_{0}}{3}-\frac{h^{2} P_{1}}{6}\right] y_{2}+\frac{h^{2} \alpha_{1} P_{N}}{6} y_{N-2} \\
& -\frac{2 h^{2} \alpha_{1} P_{N}}{3} y_{N-1}+h^{2} \alpha_{1}\left[\frac{P_{N}}{2}+\frac{2 h}{\ell^{2}}+\frac{h Q_{N}}{3}\right] y_{N}+\frac{2 h^{3}\left(1-\alpha_{1}\right)}{3} R_{0}+\frac{h^{3}}{3} R_{1}-\frac{h^{3} \alpha_{1}}{3} R_{N} .
\end{aligned}
$$

Replacing $R_{i}=y^{\prime \prime}\left(x_{i}\right)+P_{i} y^{\prime}\left(x_{i}\right)+Q_{i} y_{i}$ for $i=0,1, N$ in (4.7) and after simplifying, we obtain

$$
\begin{aligned}
T_{0}(h)= & \frac{2 h^{3} \alpha_{1}}{\ell^{2}}\left(y_{N}-y_{0}\right)+\alpha_{1} h^{2} P_{N}\left[\frac{y_{N-2}+3 y_{N}-4 y_{N-1}}{6}-\frac{h}{3} y^{\prime}\left(x_{N}\right)\right]+\frac{2 h^{3}\left(1-\alpha_{1}\right)}{3} y^{\prime \prime}\left(x_{0}\right) \\
& -\frac{h^{3}}{3} \alpha_{1} y^{\prime \prime}\left(x_{N}\right)+\left[h\left(3 a \alpha_{1}-1\right)+h^{2}\left(1-\alpha_{1}\right) P_{0}+\frac{h^{2} P_{1}}{6}\right] y_{0}+\left[2 h\left(1-2 a \alpha_{1}\right)\right. \\
& \left.-\frac{4 h^{2}\left(1-\alpha_{1}\right) P_{0}}{3}\right] y_{1}+\left[-\frac{h^{2} P_{1}}{6}-h\left(1-a \alpha_{1}\right)+\frac{h^{2}\left(1-\alpha_{1}\right) P_{0}}{3}\right] y_{2} \\
& +\frac{2 h^{3}\left(1-\alpha_{1}\right) P_{0}}{3} y^{\prime}\left(x_{0}\right)+\frac{h^{3}}{3}\left[y^{\prime \prime}\left(x_{1}\right)+P_{0} y^{\prime}\left(x_{1}\right)\right] .
\end{aligned}
$$

Using the Taylor series expansion for $y\left(x_{1}\right), y\left(x_{2}\right), y^{\prime}\left(x_{1}\right), y^{\prime \prime}\left(x_{1}\right)$ about the point $x_{0}$ and using

$$
\frac{y_{N-2}+3 y_{N}-4 y_{N-1}}{2 h}=y^{\prime}\left(x_{N}\right)-\frac{h^{2}}{3} y^{\prime \prime \prime}\left(x_{N}\right)+\cdots .
$$

in (4.8), we obtain

$$
\begin{aligned}
T_{0}(h)= & \frac{2 h^{3} \alpha_{1}}{\ell^{2}}\left(y_{N}-y_{0}\right)+\alpha_{1} h^{2} P_{N}\left[-\frac{h^{3}}{9} y^{\prime \prime \prime}\left(x_{N}\right)+\cdots\right]-\frac{h^{3} \alpha_{1}}{3} y^{\prime \prime}\left(x_{N}\right) \\
& +h^{3}\left[\frac{P_{0}-P_{1}}{3}-\frac{2 \alpha_{1}}{\ell}\right] y^{\prime}\left(x_{0}\right)+h^{4}\left[\frac{P_{0}-P_{1}}{3}-\frac{2 \alpha_{1}}{\ell}\right] y^{\prime \prime}\left(x_{0}\right)+\cdots
\end{aligned}
$$

Clearly, we can write $\left|T_{0}(h)\right| \leq C_{0} h^{3}$, where $C_{0}$ is a constant. Thus, $T_{0}(h)=\mathcal{O}\left(h^{3}\right)$ as $h \rightarrow 0$. Since $\left|\alpha_{i}\right|<a^{2}=\frac{h^{2}}{\ell^{2}}$, it is possible to get $T_{0}(h)=\mathcal{O}\left(h^{5}\right)$ as $h \rightarrow 0$ under the assumption $\left|P_{0}-P_{1}\right|=\mathcal{O}\left(h^{2}\right)$.

We get the truncation error $T_{N}(h)$ associated with the equation given in (4.5) as

$$
\begin{aligned}
T_{N}(h)= & {\left[3 h\left(1-a \alpha_{N}\right)+h^{2}\left(1-\alpha_{N}\right) P_{N}+\frac{h^{2} P_{N-1}}{6}-2 h+\frac{2 h^{3} \alpha_{N}}{\ell^{2}}-\frac{2 h^{3}\left(1-\alpha_{N}\right) Q_{N}}{3}\right] y_{N} } \\
& +\left[-4 h\left(1-a \alpha_{N}\right)-\frac{4 h^{2}\left(1-\alpha_{N}\right) P_{N}}{3}+2 h+\frac{h^{3} Q_{N-1}}{3}\right] y_{N-1}+\left[h\left(1-a \alpha_{N}\right)\right. \\
& \left.+\frac{h^{2}\left(1-\alpha_{N}\right) P_{N}}{3}-\frac{h^{2} P_{N-1}}{6}\right] y_{N-2}-\frac{h^{2} \alpha_{N} P_{0}}{6} y_{2}+\frac{2 h^{2} \alpha_{N} P_{0}}{3} y_{1}+h^{2} \alpha_{N}\left[\frac{P_{0}}{2}-\frac{2 h}{\ell^{2}}\right. \\
& \left.-\frac{h Q_{N}}{3}\right] y_{0}-\frac{2 h^{3}\left(1-\alpha_{N}\right)}{3} R_{N}-\frac{h^{3}}{3} R_{N-1}+\frac{h^{3} \alpha_{N}}{3} R_{0} .
\end{aligned}
$$

Substituting $R_{i}=y^{\prime \prime}\left(x_{i}\right)+P_{i} y^{\prime}\left(x_{i}\right)+Q_{i} y_{i}$ for $i=0, N-1, N$ in (4.7) and after simplifying, we obtain

$$
\begin{aligned}
T_{N}(h)= & \frac{2 h^{3} \alpha_{N}}{\ell^{2}}\left(y_{N}-y_{0}\right)+\alpha_{N} h^{2} P_{0}\left[\frac{-y_{2}-3 y_{0}+4 y_{1}}{6}+\frac{h}{3} y^{\prime}\left(x_{0}\right)\right]-\frac{2 h^{3}\left(1-\alpha_{N}\right)}{3} y^{\prime \prime}\left(x_{N}\right) \\
& +\frac{h^{3}}{3} \alpha_{N} y^{\prime \prime}\left(x_{0}\right)+\left[-h\left(3 a \alpha_{N}-1\right)+h^{2}\left(1-\alpha_{N}\right) P_{N}+\frac{h^{2} P_{N-1}}{6}\right] y_{N}+\left[-2 h\left(1-2 a \alpha_{1}\right)\right.
\end{aligned}
$$




$$
\begin{aligned}
& \left.-\frac{4 h^{2}\left(1-\alpha_{N}\right) P_{N}}{3}\right] y_{N-1}+\left[-\frac{h^{2} P_{N-1}}{6}+h\left(1-a \alpha_{N}\right)+\frac{h^{2}\left(1-\alpha_{N}\right) P_{N}}{3}\right] y_{N-2} \\
& -\frac{2 h^{3}\left(1-\alpha_{N}\right)}{3} y^{\prime}\left(x_{N}\right)-\frac{h^{3}}{3}\left[y^{\prime \prime}\left(x_{N-1}\right)+P_{N} y^{\prime}\left(x_{N-1}\right)\right] .
\end{aligned}
$$

Using the Taylor series expansion for $y\left(x_{N-1}\right), y\left(x_{N}\right), y^{\prime}\left(x_{N-1}\right), y^{\prime \prime}\left(x_{N-1}\right)$ about the point $x_{N}$ and using

$$
\frac{y_{2}+3 y_{0}-4 y_{1}}{2 h}=y^{\prime}\left(x_{0}\right)-\frac{h^{2}}{3} y^{\prime \prime \prime}\left(x_{0}\right)+\cdots .
$$

in (4.10), we obtain

$$
\begin{aligned}
T_{N}(h)= & \frac{2 h^{3} \alpha_{N}}{\ell^{2}}\left(y_{N}-y_{0}\right)+\alpha_{N} h^{2} P_{0}\left[\frac{h^{3}}{9} y^{\prime \prime \prime}\left(x_{0}\right)-\cdots\right]+\frac{h^{3} \alpha_{N}}{3} y^{\prime \prime}\left(x_{0}\right) \\
& h^{3}\left[\frac{P_{N-1}-P_{N}}{3}-\frac{2 \alpha_{N}}{\ell}\right] y^{\prime}\left(x_{N}\right)+h^{4}\left[\frac{P_{N-1}-P_{N}}{3}-\frac{2 \alpha_{N}}{\ell}\right] y^{\prime \prime}\left(x_{N}\right)+\cdots
\end{aligned}
$$

From (4.11), we can write $\left|T_{N}(h)\right| \leq C_{N} h^{3}$, where $C_{N}$ is a suitable constant. Thus, $T_{N}(h)=\mathcal{O}\left(h^{3}\right)$ as $h \rightarrow 0$. Since $\left|\alpha_{i}\right|<a^{2}=\frac{h^{2}}{\ell^{2}}$, it is possible to get $T_{N}(h)=\mathcal{O}\left(h^{5}\right)$ as $h \rightarrow 0$ under the assumption $\left|P_{N-1}-P_{N}\right|=\mathcal{O}\left(h^{2}\right)$.

Further, we can write the truncation error $T_{i}(h)$ associated with the equation given in (4.6) as

$$
\begin{aligned}
T_{i}(h)= & {\left[12+2 h^{2} Q_{i-1}-3 h P_{i-1}-4 h+h P_{i+1}\right] y_{i-1}+\left[8 h^{2} Q_{i}-24+4 h P_{i-1}\right] y_{i}+[12} \\
& \left.+2 h^{2} Q_{i-1}-h P_{i-1}+4 h+3 h P_{i+1}\right] y_{i+1}+\left[2 h^{2}\left(\alpha_{i}+2 \alpha_{i+1}\right) Q_{0}-\frac{12 h^{2}}{\ell^{2}}\left(\alpha_{i+1}-\alpha_{i}\right)\right. \\
& \left.-\frac{18 h \alpha_{i+1}}{\ell}-3 h\left(\alpha_{i}+2 \alpha_{i+1}\right) P_{0}\right] y_{0}+\left[\frac{24 h \alpha_{i+1}}{\ell}+4 h\left(\alpha_{i}+2 \alpha_{i+1}\right) P_{0}\right] y_{1}+\left[-\frac{6 h \alpha_{i+1}}{\ell}\right. \\
& \left.-2 h\left(\alpha_{i}+2 \alpha_{i+1}\right) P_{0}\right] y_{2}+\left[-\frac{6 h \alpha_{i}}{\ell}-2 h\left(2 \alpha_{i}+\alpha_{i+1}\right) P_{N}\right] y_{N-2}+\left[4 h\left(2 \alpha_{i}+\alpha_{i+1}\right) P_{N}\right. \\
& \left.+\frac{24 h \alpha_{i}}{\ell}\right] y_{N-1}+\left[-2 h^{2}\left(2 \alpha_{i}+\alpha_{i+1}\right) Q_{N}+\frac{12 h^{2}}{\ell^{2}}\left(\alpha_{i+1}-\alpha_{i}\right)-3 h\left(2 \alpha_{i}+\alpha_{i+1}\right) P_{N}\right. \\
& \left.-\frac{18 h \alpha_{i}}{\ell}\right] y_{N}-2 h^{2} R_{i-1}-8 h^{2} R_{i}-2 h^{2} R_{i+1}-2 h^{2}\left(\alpha_{i}+2 \alpha_{i+1}\right) R_{0}+2 h^{2}\left(2 \alpha_{i}+\alpha_{i+1}\right) R_{N} .
\end{aligned}
$$

Replacing $R_{j}=y^{\prime \prime}\left(x_{j}\right)+P_{j} y^{\prime}\left(x_{j}\right)+Q_{j} y_{j}$ for $j=0, i-1, i, i+1, N$ and using the Taylor series expansions of $y\left(x_{i}\right)$ and $y\left(x_{i+1}\right)$ around the point $x_{i-1}$, we obtain the following:

$$
\begin{aligned}
T_{i}(h)= & 4 h^{3}\left(1-2 P_{i}\right) y^{\prime \prime}\left(x_{i-1}\right)+h^{3}\left[12-\frac{8 h}{3}-\frac{2 h P_{i-1}}{3}-\frac{4 h^{2} P_{i}}{3}+\frac{10 h P_{i+1}}{3}\right] y^{\prime \prime \prime}\left(x_{0}\right)+\cdots \\
& +\left[2 h^{2}\left(\alpha_{i}+2 \alpha_{i+1}\right) Q_{0}-\frac{12 h^{2}}{\ell^{2}}\left(\alpha_{i+1}-\alpha_{i}\right)-\frac{18 h \alpha_{i+1}}{\ell}-3 h\left(\alpha_{i}+2 \alpha_{i+1}\right) P_{0}\right] y_{0} \\
& +\left[4 h\left(\alpha_{i}+2 \alpha_{i+1}\right) P_{0}+\frac{24 h \alpha_{i+1}}{\ell}\right] y_{1}+\left[-\frac{6 h \alpha_{i+1}}{\ell}-2 h\left(\alpha_{i}+2 \alpha_{i+1}\right) P_{0}\right] y_{2} \\
& +\left[-2 h\left(2 \alpha_{i}+\alpha_{i+1}\right) P_{N}-\frac{6 h \alpha_{i}}{\ell}\right] y_{N-2}+\left[4 h\left(2 \alpha_{i}+\alpha_{i+1}\right) P_{N}+\frac{24 h \alpha_{i}}{\ell}\right] y_{N-1} \\
& +\left[-2 h^{2}\left(2 \alpha_{i}+\alpha_{i+1}\right) Q_{N}+\frac{12 h^{2}}{\ell^{2}}\left(\alpha_{i+1}-\alpha_{i}\right)-3 h\left(2 \alpha_{i}+\alpha_{i+1}\right) P_{N}-\frac{18 h \alpha_{i}}{\ell}\right] y_{N}
\end{aligned}
$$

Since $\left|\alpha_{i}\right|<a^{2}=\frac{h^{2}}{\ell^{2}}$, we have $\left|T_{i}(h)\right| \leq C_{i} h^{3}$, where $C_{i}$ is a constant. Thus, $T_{i}(h)=\mathcal{O}\left(h^{3}\right)$ as $h \rightarrow 0$ for $i=1,2, \ldots, N-1$. Consequently, any approximation solution will converge to the exact solution as $h \rightarrow 0$.

\section{Numerical results and discussion}

First, we consider the case (a) when $y^{\prime}$ is absent. Here, we try to get a smooth fractal solution of the following BVP:

Example 5.1. Consider the two point boundary value problem

$$
y^{\prime \prime}+y+1=0, \quad y(0)=y(1)=0,
$$




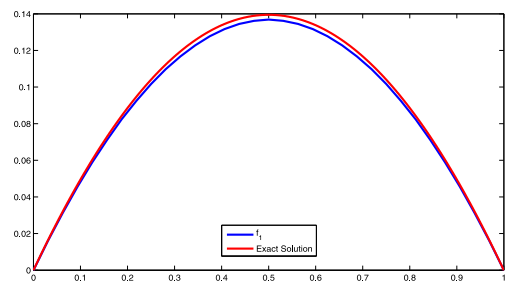

(a) Exact solution $S$, fractal solution $f_{1}$

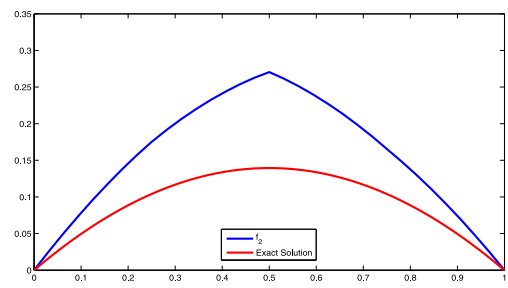

(b) Exact solution $S$, fractal solution $f_{2}$

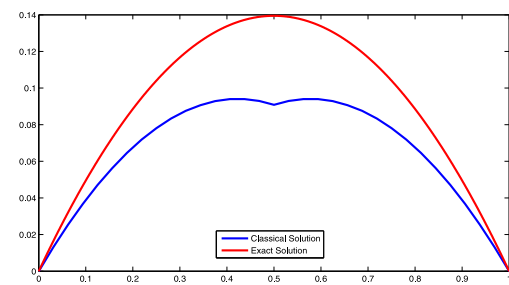

(c) Exact solution $S$, classical solution $P$

Fig. 1. Solutions of BVP with different scaling factors.

whose analytical (or exact) solution is

$$
y(x)=\cos x+\tan (1 / 2) \sin x-1 .
$$

Divide the interval $[0,1]$ into two equal sub-intervals $(N=2)$ as $[0,1 / 2]$ and $[1 / 2,1]$ with $x_{0}=0, x_{1}=1 / 2$ and $x_{2}=1$. It is clear that $h_{1}=h_{2}=h=1 / 2, \ell=1$ and $a=1 / 2$. Assume the scaling factors $\alpha_{i}=0.1=\alpha, i=1,2$. Here $P(x)=1$, $Q(x)=0$, and $R(x)=-1$. Thus, $P_{j}=1, Q_{j}=0$, and $R_{j}=-1$ for $j=0,1,2$. From (3.17), we will get the following equation for $i=1$ :

$$
\alpha\left(d_{2}-d_{0}\right)+8 y_{1}-\frac{2}{3} y_{1}=-\alpha+1 \Rightarrow y_{1}=\frac{3 \alpha}{22}\left(d_{0}-d_{2}-1\right)+\frac{3}{22} .
$$

From (3.6) and (3.7), we find the following equations respectively:

$$
\begin{aligned}
& d_{0}=f^{\prime}(0)=\frac{\alpha}{88}\left(69 d_{0}-25 d_{2}-49\right)+\frac{47}{88}, \\
& d_{2}=f^{\prime}(1)=\frac{\alpha}{88}\left(69 d_{2}-25 d_{0}+49\right)-\frac{47}{88} .
\end{aligned}
$$

Solving the above system (5.2)-(5.3), we compute $d_{0}=-d_{2}=0.5356$ and $y_{1}=y(1 / 2)=0.136904 \equiv 3 / 22$. Now, we will compute the moments using the above values with $y_{0}=y_{2}=0$.

$$
\begin{aligned}
& M_{0}=R_{0}-P_{0} y_{0} \Rightarrow M_{0}=-1, \\
& M_{2}=R_{2}-P_{2} y_{2} \Rightarrow M_{2}=-1, \\
& M_{1}=R_{1}-P_{1} y_{1} \Rightarrow M_{1}=-1.1081 .
\end{aligned}
$$

These moments are then used in (3.1) to write the functional equation of a cubic spline FIF (say $\left.f_{1}\right)$ in the following:

$$
f_{1}\left(L_{i}(x)\right)= \begin{cases}\frac{1}{40} f_{1}(x)-\frac{229}{40000} x^{3}-\frac{81}{160} x^{2}+\frac{1}{80} x & \text { for } x \in[0,1 / 2], \\ \frac{1}{40} f_{1}(x)+\frac{229}{40000} x^{3}-\frac{5187}{40000} x^{2}-\frac{269}{20000} x+\frac{687}{5000}, & \text { for } x \in[1 / 2,1] .\end{cases}
$$

The iteration of this functional equation gives us the graph of the desired cubic spline FIF in Fig. 1(a). By assuming different values to scaling factors, say $\alpha_{1}=1 / 8$ and $\alpha_{2}=-1 / 7$, we obtain the following system of equations with the unknown variables $y_{1}, d_{0}, d_{2}$ directly from (3.20)-(3.22):

$$
\begin{aligned}
& y_{1}+0.0195 d_{0}+0.0170 d_{3}=0.2740 \\
& y_{1}-0.45 d_{0}=0.1050 \\
& y_{1}+0.5450 d_{2}=0.1491
\end{aligned}
$$

The solution of the above system gives $y_{1}=0.2705, d_{0}=0.3678$ and $d_{2}=-0.2172$. Thus, we get $M_{0}=-1$, $M_{1}=-1.2705$ and $M_{2}=-1$. Using these moments in (3.1), we obtain another cubic spline FIF (say $f_{2}$ ), see Fig. 1(b), and its functional equation is

$$
f_{2}\left(L_{i}(x)\right)= \begin{cases}\frac{1}{32} f_{2}(x)-0.0113 x^{3}-0.1094 x^{2}+0.3911 x, & \text { for } x \in[0,1 / 2], \\ -\frac{1}{28} f_{2}(x)+0.0113 x^{3}-0.1767 x^{2}-0.0105 x+0.2705, & \text { for } x \in[1 / 2,1]\end{cases}
$$

Note that the scaling factors can be chosen from the range $\left(-a^{2}, a^{2}\right)=(-0.25,0.25)$. We have shown $f_{2}$ to demonstrate the possible of getting a large class of solutions by fractal methodology. By setting all scaling factors to zero, we obtain the classical cubic spline interpolant $P$, see Fig. 1(c). From Fig. 1, it is easy to observe that the fractal cubic spline $f_{1}$ is a better approximant in comparison with the classical cubic spline solution. 


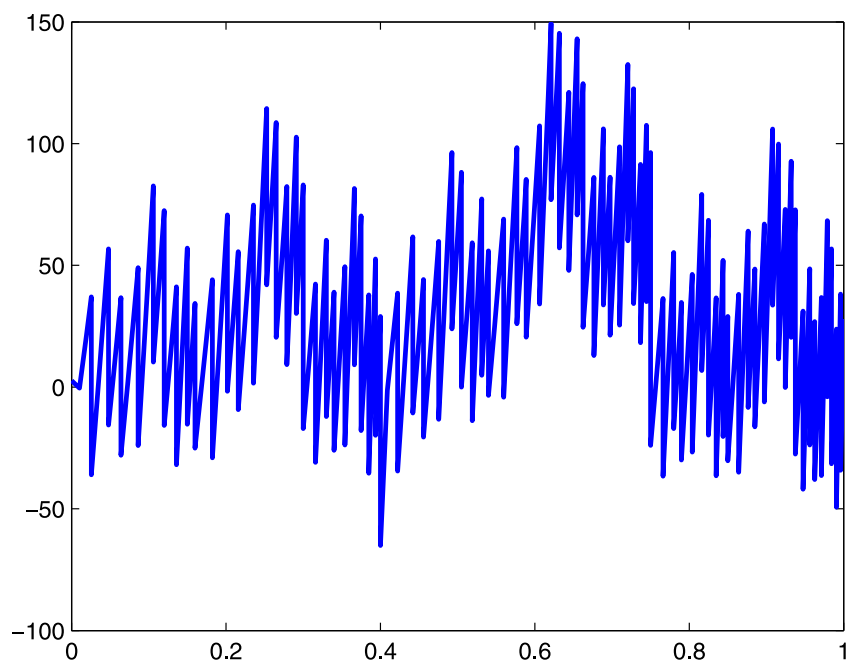

Fig. 2. Nowhere differentiable curve $R(x)$.

Table 1

Parameters for cubic spline fractal solutions of BVP (5.4).

\begin{tabular}{ll}
\hline Fig. & Scaling vector, end derivatives and moments \\
\hline 3a & $\alpha=(0.8,0.8,0.8), d_{0}=9.4232, d_{3}=19.4085$ \\
& $M_{0}=1.9987, M_{1}=-65.0177, M_{2}=93.8429, M_{3}=4.9980$ \\
3b & $\alpha=(0.8,0.8,0.8), d_{0}=9.4232, d_{3}=19.4085$ \\
& $M_{0}=1.7445, M_{1}=-64.8296, M_{2}=93.8429, M_{3}=4.9667$ \\
3c & $\alpha=(0.35,0.3,0.25), d_{0}=6.8810, d_{3}=19.3810$ \\
& $M_{0}=9.1776, M_{1}=-54.8010, M_{2}=99.3891, M_{3}=35.9852$ \\
3d & $\alpha=(0.8,0.8,0.8), d_{0}=9.4232, d_{3}=19.4085$ \\
& $M_{0}=-45.1382, M_{1}=-28.7917, M_{2}=74.3697, M_{3}=62.6486$ \\
3e & $\alpha=(0.35,0.3,0.25), d_{0}=6.8810, d_{3}=19.3810$ \\
3f & $M_{0}=9.1776, M_{1}=-54.8010, M_{2}=99.3891, M_{3}=35.9852$ \\
& $\alpha=(0,0,0), d_{0}=9.4232, d_{3}=19.4085$ \\
& $M_{0}=-28.3648, M_{1}=-47.1184, M_{2}=93.5364, M_{3}=42.1338$ \\
\hline &
\end{tabular}

Example 5.2. Consider the following two-point BVP

$$
y^{\prime \prime}+P(x) y^{\prime}+Q(x) y=R(x), \quad y(0)=1, y(1)=2,
$$

where $P(x)=x^{2}+0.1, Q(x)=\sin x+0.1$ and $R(x)$ is the nowhere differentiable function as shown in Fig. 2.

Divide the interval $[0,1]$ into subintervals as $\left[x_{0}, x_{1}\right],\left[x_{1}, x_{2}\right]$ and $\left[x_{2}, x_{3}\right]$, where $x_{0}=0, x_{1}=0.4, x_{2}=0.75$ and $x_{3}=1$. From the graph, it is found that $R_{0}=2.6868, R_{1}=-65.0670, R_{2}=96.3384$ and $R_{3}=27.9332$ at the knot points. Thus from (3.6)-(3.7) and (3.11)-(3.17), we obtain a system of equations with the unknown variables $y_{1}, y_{2}, d_{0}$ and $d_{3}$. Assuming the scaling factors as $\alpha_{i}=0.8, i \in \mathbb{N}_{3}$, after some simple algebraic computations, we obtain the values $d_{0}=9.4232, d_{3}=19.4085$ and the moments $M_{0}=1.7445, M_{1}=-64.8296, M_{2}=93.8429$ and $M_{3}=4.9667$. Using these values, we have calculated $y_{1}=1$ and $y_{2}=-1$.

The exact solution $S$ of the BVP is given in Fig. 3a. An approximated fractal cubic spline solution using moments is as shown in Fig. $3 \mathrm{~b}$ and is defined as follows:

$$
f_{1}\left(L_{i}(x)\right)=\left\{\begin{array}{lr}
\frac{16}{125} f_{1}(x)-\frac{4669}{2500} x^{3}+\frac{4}{125} x^{2}+\frac{129}{50} x, & \text { for } x \in[0,0.4], \\
\frac{49}{500} f_{1}(x)+\frac{5491}{2500} x^{3}-\frac{2059}{500} x^{2}+\frac{32}{625} x-\frac{110}{625}, & \text { for } x \in[0.4,0.75], \\
\frac{1}{20} f_{1}(x)-\frac{235}{250} x^{3}+\frac{715}{250} x^{2}-\frac{91}{100} x+\frac{91}{100}, & \text { for } x \in[0.75,1] .
\end{array}\right.
$$

Similarly, for different choices of scaling factors $\alpha_{i}, i=1,2,3$, we obtain different values for $d_{0}, d_{3}, M_{0}, M_{1}, M_{2}$ and $M_{3}$ which are listed in Table 1 . With respect to these values, we obtain various fractal cubic spline solutions, and they are presented in Figs. 3c-e. Setting all scaling factors to 0, we obtain the classical cubic spline solution $P$ defined through moments as shown in Fig. 3f. A comparison between the exact solution, 4 different cubic fractal solutions and the classical solution are illustrated in Fig. 4. The uniform errors between the exact solution of the BVP with various fractal cubic spline solutions are listed in Table 2. It is easy to observe from Fig. 4 that the cubic fractal spline solution $f_{1}$ is the closest approximated solution among the proposed five solutions to the given BVP, whose non-homogeneous term is nowhere differentiable function in the given domain. 


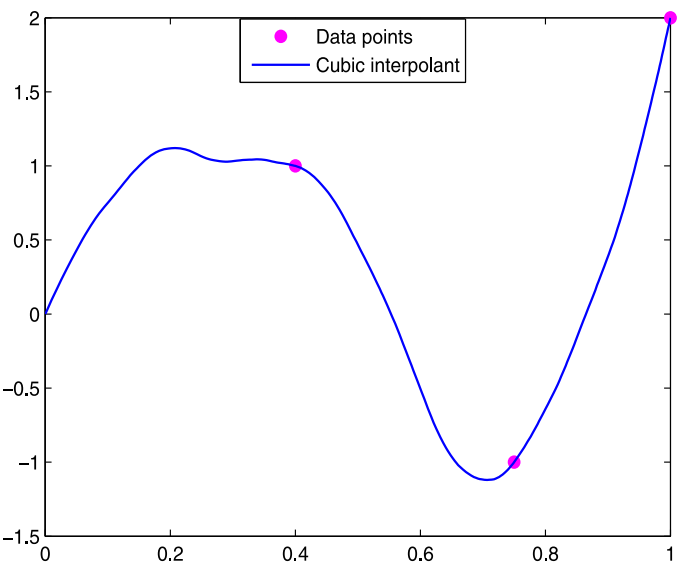

(a) Exact solution : $S$

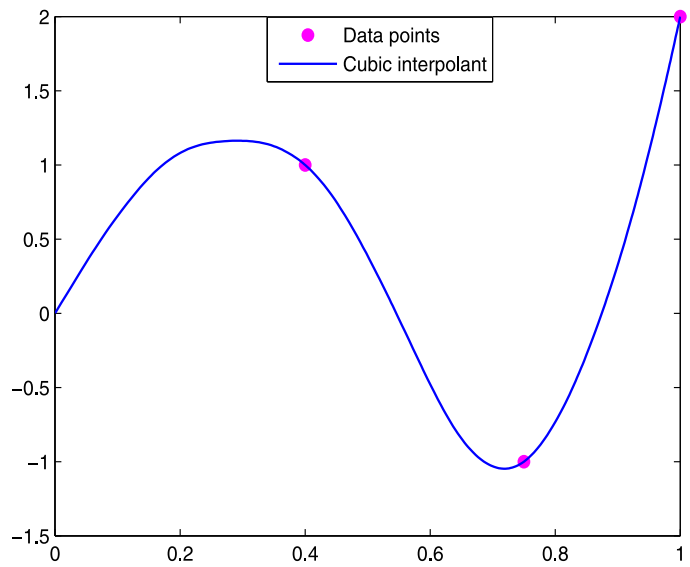

(c) Cubic fractal solution: $f_{2}$

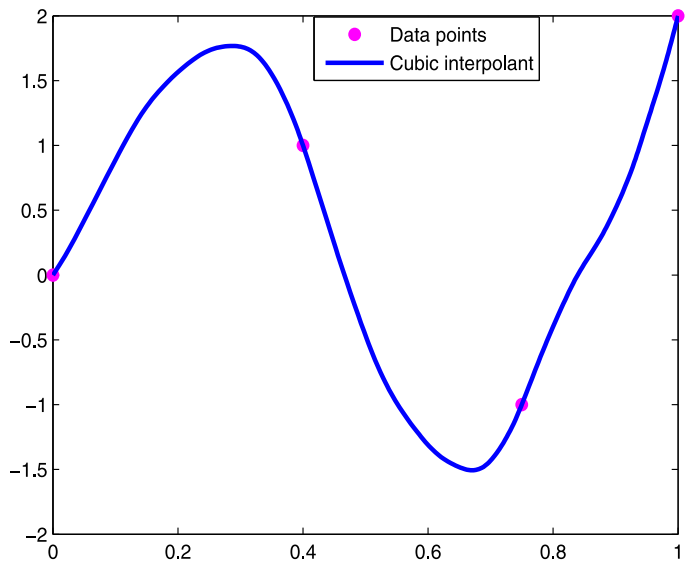

(e) Cubic fractal solution : $f_{4}$

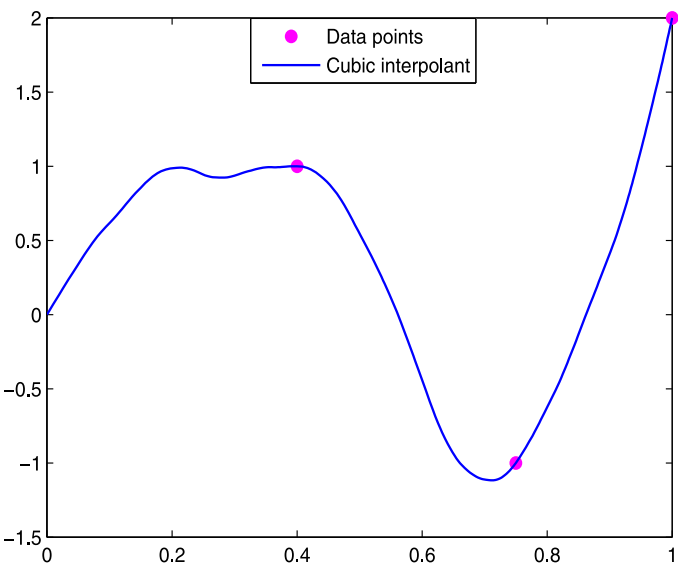

(b) Cubic fractal solution : $f_{1}$

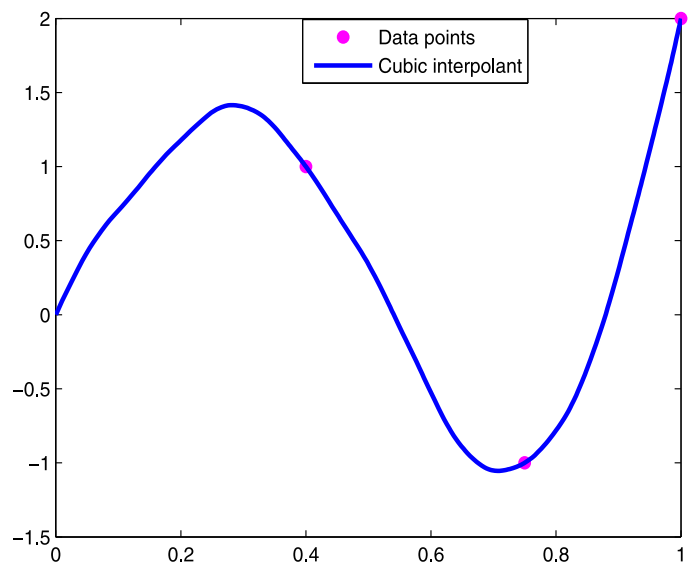

(d) Cubic fractal solution: $f_{3}$

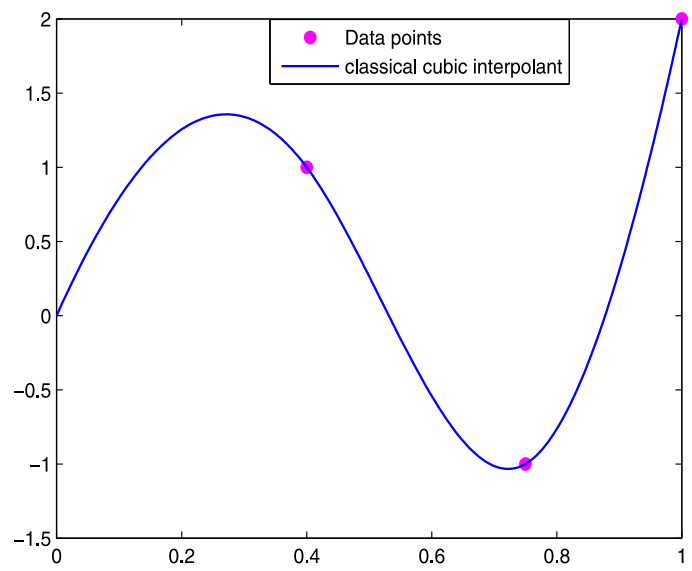

(f) Cubic classical solution : $P$

Fig. 3. Solutions of BVP with different scaling factors.

Remark 5.1. Note that the solution of BVP (3.8)-(3.9) is not unique if $y(x)$ is not infinitely differentiable. When we assume that $y^{\prime \prime}$ is not differentiable, it is possible to get a wide variety of solutions by fractal methodology due to the flexibility offered by the scaling factors. The flexibility in the choice of fractal interpolants can be harvested to elect a 


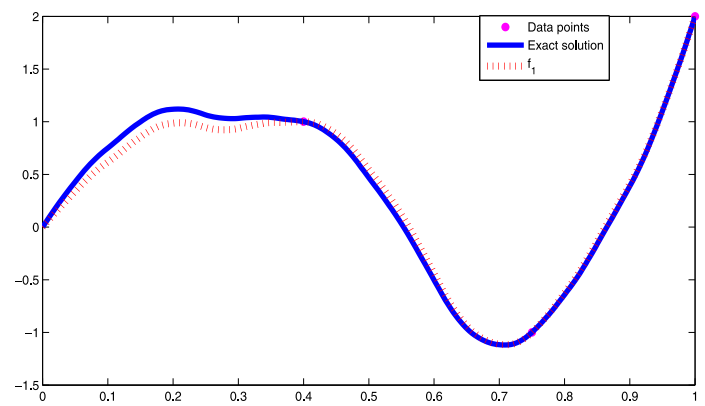

(a) $S, f_{1}$

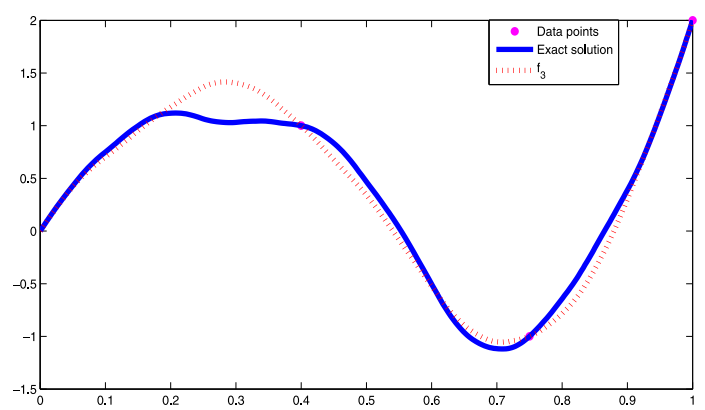

(c) $S, f_{3}$

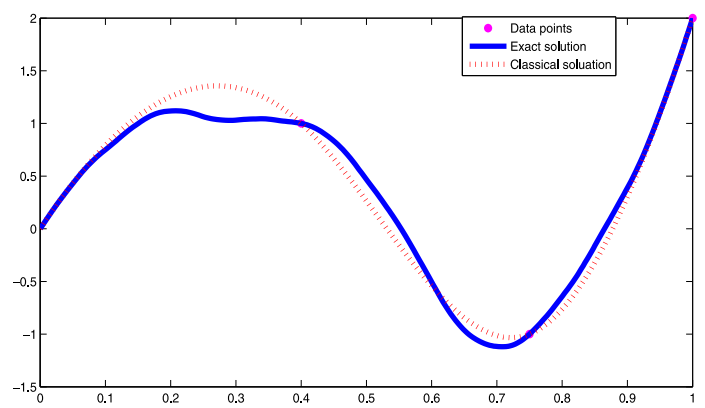

(e) $S, P$

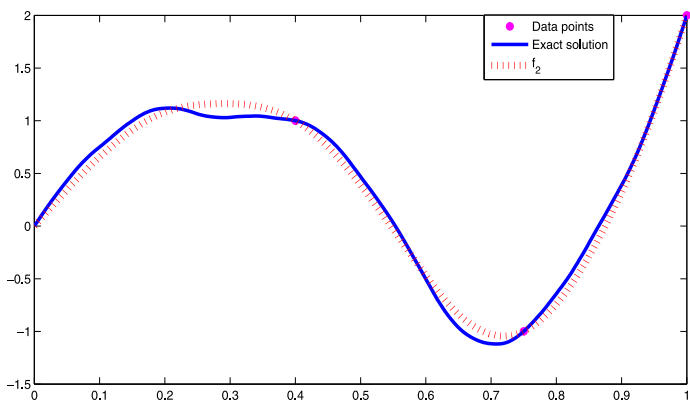

(b) $S, f_{2}$

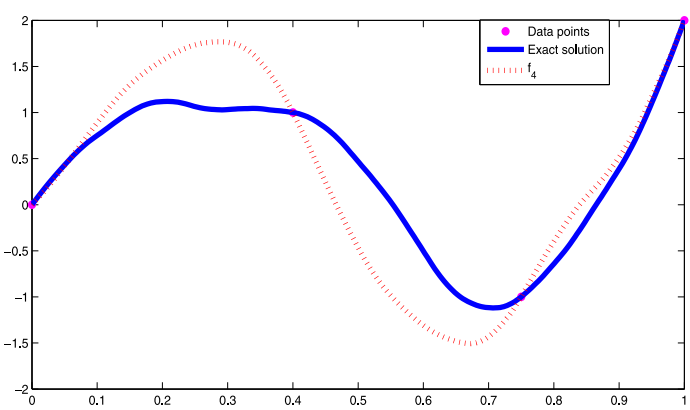

(d) $S, f_{4}$

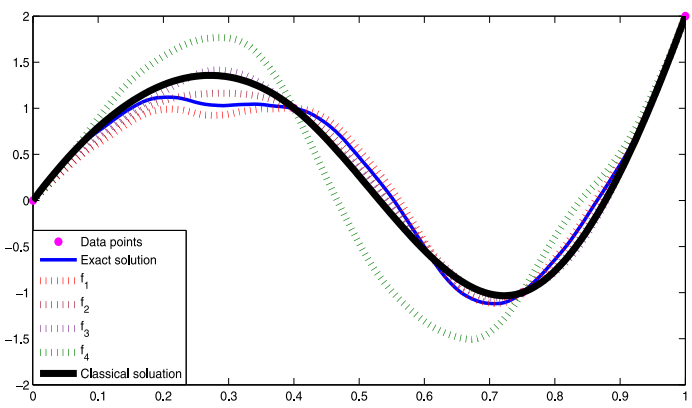

(f) $S, f_{1}, f_{2}, f_{3}, f_{4}, P$

Fig. 4. Approximation of exact solution of BVP (5.4) with the proposed cubic spline FIFs.

Table 2

Uniform error between the exact solution and cubic spline fractal solutions.

\begin{tabular}{ll}
\hline$\left\|S-f_{1}\right\|_{\infty}$ & 0.1401 \\
$\left\|S-f_{2}\right\|_{\infty}$ & 0.1445 \\
$\left\|S-f_{3}\right\|_{\infty}$ & 0.3846 \\
$\left\|S-f_{4}\right\|_{\infty}$ & 1.03 \\
$\|S-C\|_{\infty}$ & 0.3232 \\
\hline
\end{tabular}

suitable interpolant by concentrating some desirable features such as smoothness, fairness, fractality in the derivative, and threshold error between $R(x)$ and its approximation. The question on "optimum curve" can be addressed based on Levkovich's work [23]. Here the problem is for given functions $P(x), Q(x), R(x)$, find the IFS parameters of $y$ such that $y^{\prime \prime}+P y^{\prime}+Q=R$ on $\left[x_{0}, x_{N}\right]$. In Levkovich's work, contraction affine mappings generating a given function is obtained based on the connection between the maxima skeleton of wavelet transform of the function and positions of the fixed points of the affine mappings. One can adapt a similar procedure to find the connection between the strongest singularities 
of $R(x)$ and the 2nd derivative of the fixed points of the cubic spline IFS. This will give an idea to fix the position of grid points on $\left[x_{0}, x_{N}\right]$. Then, the optimal curve can be defined as the fractal curve produced by a specific set of parameters that minimizes a suitable numerical quantity assigned to all possible curves obtained by the scheme ensuring that the error $\left\|y^{\prime \prime}+P y^{\prime}+Q-R\right\|$ is minimal. Such a constrained optimization problem may be solved by means of a differential evolution optimization algorithm/genetic algorithm to choose the scaling parameters for construction of the desired cubic spline FIF.

\section{Summary}

In this work, we have discussed the solutions of two-point BVPs by using cubic spline FIFs through moments in a deterministic manner even if the non-homogeneous differential equation involves a continuous function which is nowhere differentiable. Using the continuity condition at the grid points of the cubic spline fractal and the end point conditions by the derivatives, we have computed the values at the nodes $y_{1}, y_{2}, \ldots, y_{N-1}$ from a tridiagonal system. Then, the moments are computed in terms of $y_{i}, i \in \mathbb{N}_{N} \cup\{0\}$ to obtain the desired cubic spline fractal solution. Selecting the scaling factors to be zero and equal interpolating knot sequences, the proposed method coincides with the solution developed in [11]. Hence, our method is more general than the classical cubic spline solution of the second order ordinary BVP. The truncation error of the proposed method is $\mathcal{O}\left(h^{3}\right)$ as $h \rightarrow 0$.

\section{Acknowledgments}

The first author is thankful for the project: MTR/2017/000574 - MATRICS from the Science and Engineering Research Board (SERB), Government of India. The authors would like to thank the anonymous referees for their valuable suggestions to improve the exposition of the paper.

\section{References}

[1] M.F. Barnsley, Fractals Everywhere, Academic Press, Orlando, Florida, 1988.

[2] M.F. Barnsley, A.N. Harrington, The calculus of fractal interpolation functions, J. Approx. Theory 57 (1) (1989) 14-34.

[3] A.K.B. Chand, G.P. Kapoor, Generalized cubic spline fractal interpolation functions, SIAM J. Numer. Anal. 44 (2) (2006) 655-676.

[4] A.K.B. Chand, M.A. Navascués, Generalized Hermite fractal interpolation, Rev. R. Acad. Cienc. Exactas Fís. Quím. Nat. Zaragoza (2) 64 (2) (2009) $107-120$.

[5] A.K.B. Chand, M.A. Navascués, Natural bicubic spline fractal interpolation, Nonlinear Anal. TMA 69 (2008) 3679-3691.

[6] A.K.B. Chand, N. Vijender, M.A. Navascués, Shape preservation of scientific data through rational fractal splines, Calcolo 51 (2) (2014) 329-362.

[7] A.K.B. Chand, K.R. Tyada, Constrained shape preserving rational cubic fractal interpolation functions, Rocky Mountain J. Math. 48 (1) (2018) 75-105.

[8] M.A. Navascués, Fractal polynomial interpolation, Z. Anal. Anwend. 24 (2) (2005) 401-418.

[9] M.A. Navascués, M.V. Sebastián, Smooth fractal interpolation, J. Inequal. Appl. (2006) 1-20, Art. ID 78734.

[10] W.G. Bickley, Piecewise cubic interpolation and two-point boundary problems, Comput. J. 11 (2) (1968) 206-208.

[11] E.L. Albasiny, W.D. Hoskins, Cubic spline solution to two-point boundary value problems, Comput. J. 12 (2) (1969) $151-153$.

[12] D.J. Fyfe, The use of cubic splines in the solution of two-point boundary value problems, Comput. J. 12 (2) (1969) $188-192$.

[13] M.K. Jain, T. Aziz, Spline function approximation for differential equations, Comput. Methods Appl. Mech. Engrg. 26 (1981) 129-143.

[14] E.A. Al-Said, Cubic spline method for solving two-point boundary value problems, Korean J. Comput. Appl. Math. 5 (3) (1998) 669-680.

[15] H. Ramos, M.A. Rufai, A third-derivative two-step block Falkner-type method for solving general second-order boundary-value systems, Math. Comput. Simulation 165 (2019) 139-155.

[16] H. Ramos, M.A. Rufai, Numerical solution of boundary value problems by using an optimized two-step block method, Numer. Algorithms 84 (2020) 229-251.

[17] Mark I. Modebeia, S.N. Jatorb, H. Ramos, Block hybrid method for the numerical solution of fourth order boundary value problems, Comput Appl. Math. 377 (2020) Article No. 112876.

[18] A. Atangana, Fractional discretization: The African's tortoise walk, Chaos Solitons Fractals 130 (2020) 109399, 24 pp.

[19] A. Jayaraman, A. Belmonte, Oscillations of a solid sphere falling through a wormlike micellar fluid, Phys. Rev. 67 (2003) 65301.

[20] W.M. Siebert, Circuits, Signals, and Systems, MIT press, Cambridge, 1986.

[21] M.F. Barnsley, Fractal functions and interpolations, Constr. Approx. 2 (1986) 303-329.

[22] H.B. Keller, Numerical Methods for Two-Point Boundary Value Problems, Blaisdell, Waltham, 1968.

[23] L.I. Levikovich, Wavelet-based determination of generating matrices for fractal interpolation functions, Regul. Chaotic Dyn. 3 (2) (1998) 20-29. 